\title{
ATMRSIALCL
}

\section{PROPERTIES OF THE OPTIMAL TRAJECTORIES FOR COPLANAR, AEROASSISTED ORBITAL TRANSFER}

by

A. MIELE, T. WANG, AND A. W. DEATON

(NASA-CR-1B7733) PROPEQTIES OF THE UPTIMAL

$N 91-14366$

TRAJECTORIES FOP COPLANAR, AEROASSISTEO

ORAITAL TPANJFER (PiCOUNiv.) $58 \mathrm{pCSCL} 22 \mathrm{~A}$

Unclas

$G J / 13 \quad 0321361$

RICE UNIVERSITY 


\section{PROPERTIES OF THE OPTIMAL TRAJECTORIES FOR COPLANAR, AEROASSISTED ORBITAL TRANSFER by}

A. MIELE, T. WANG, and A. W. DEATON

RICE UNIVERSITY 
Properties of the Optimal Trajectories for Coplanar, Aeroassisted Orbital Transfer ${ }^{1}$

by
A. Miele ${ }^{2}$, T. Wang $^{3}$, and A. W. Deaton ${ }^{4}$

\footnotetext{
This research was supported by NASA Marshall space Flight Center Grant No. NAG-8-820, by Jet Propulsion Laboratory Contract No. 956415, and by Texas Advanced Technology Program Grant No. TATP-003604020.

2 Foyt Family Professor of Aerospace Sciences and Mathematical Sciences, Aero-Astronautics Group, Rice University, Houston, Texas.

${ }^{3}$ Senior Research Scientist, Aero-Astronautics Group, Rice University, Houston, Texas.

${ }^{4}$ Aerospace Engineer and Team Leader, Flight Mechanics Branch, Systems Analysis and Integration Laboratory, NASA Marshall Space Flight Center, Huntsville, Alabama.
} 
Abstract. This report is concerned with the optimization of trajectories for coplanar, aeroassisted orbital transfer (AOT) from a high Earth orbit (HEO) to a low Earth orbit (LEO). In particular, HEO can be a geosynchronous Earth orbit (GEO). It is assumed that the initial and final orbits are circular, that the gravitational field is central and is governed by the inverse square law, and that two impulses are employed, one at HEO exit and one at LEO entry. During the atmospheric pass, the trajectory is controlled via the lift coefficient in such a way that the total characteristic velocity is minimized.

First, an ideal optimal trajectory is determined analytically for lift coefficient unbounded. This trajectory is called grazing trajectory, because the atmospheric pass is made by flying at constant altitude along the edge of the atmosphere until the excess velocity is depleted. For the grazing trajectory, the lift coefficient varies in such a way that the lift, the centrifugal force due to the Earth's curvature, the weight, and the Coriolis force due to the Earth's rotation are in static balance. Also, the grazing trajectory minimizes the total characteristic velocity and simultaneously nearly minimizes the peak values of the altitude drop, the dynamic pressure, and the heating rate.

Next, starting from the grazing trajectory results, a real optimal trajectory is determined numerically for lift coefficient bounded from both below and above. This trajectory is characterized by atmospheric penetration with the smallest possible entry angle, followed by flight at the lift coefficient lower bound. 
Consistently with the grazing trajectory behavior, the real optimal trajectory minimizes the total characteristic velocity and simultaneously nearly minimizes the peak values of the altitude drop, the dynamic pressure, and the heating rate.

Key Words. Flight mechanics, astrodynamics, aeroassisted orbital transfer, optimal trajectories, nonlinear two-point boundary-value problems, sequential gradient-restoration algorithm. 


\section{Notations}

$\mathrm{C}_{\mathrm{D}}$ = drag coefficient;

$C_{L}=$ lift coefficient;

$\mathrm{D}=\mathrm{drag}, \mathrm{N}$;

DP = dynamic pressure, $\mathrm{N} / \mathrm{m}^{2}$;

$\mathrm{E} \quad=$ lift-to-drag ratio modulus;

$\mathrm{g}=$ local acceleration of gravity, $\mathrm{m} / \mathrm{sec}^{2}$;

$g_{a}=$ acceleration of gravity at $\mathrm{h}=\mathrm{h}_{\mathrm{a}}, \mathrm{m} / \mathrm{sec}^{2}$;

$\mathrm{h}$ = altitude, $\mathrm{m}$;

$\mathrm{h}_{\mathrm{a}}=$ thickness of the atmosphere, $\mathrm{m}$;

$\mathrm{HR}=$ heating rate, $\mathrm{W} / \mathrm{m}^{2}$;

$\mathrm{L}=$ Iift, $\mathrm{N}$;

$\mathrm{m}=$ mass, $\mathrm{kg}$;

$r$ = radial distance from the center of the Earth, $m$;

$r_{\mathrm{e}}=$ radius of the Earth, $\mathrm{m}$;

$r_{a}=$ radius of the outer edge of the atmosphere, $m$;

$\mathrm{S} \quad=$ reference surface area, $\mathrm{m}^{2}$;

$t \quad=\mathrm{T} / \tau=$ dimensionless time;

$\mathrm{T}=$ running time, sec;

$\mathrm{V}=$ velocity, $\mathrm{m} / \mathrm{sec}$;

$\mathrm{v}_{\mathrm{a}}=$ circular velocity at $\mathrm{r}=\mathrm{r}_{\mathrm{a}}, \mathrm{m} / \mathrm{sec} ;$

$\mathrm{v}_{*}=$ reference velocity, $\mathrm{m} / \mathrm{sec}$;

$\alpha$. = angle of attack, rad;

$\gamma=$ path inclination, rad;

$\mu=$ Earth's gravitational constant, $\mathrm{m}^{3} / \mathrm{sec}^{2}$;

$\rho=\operatorname{air}$ density, $\mathrm{kg} / \mathrm{m}^{3}$;

$\rho_{\mathrm{a}}=$ air density at $\mathrm{h}=\mathrm{h}_{\mathrm{a}}, \mathrm{kg} / \mathrm{m}^{3}$;

$\rho_{*}=$ reference air density, $\mathrm{kg} / \mathrm{m}^{3}$; 


$$
\begin{aligned}
& \tau=\text { final time, sec; } \\
& \omega=\text { Earth's angular velocity, rad/sec; } \\
& \Delta V=\text { characteristic velocity, } \mathrm{m} / \mathrm{sec} .
\end{aligned}
$$

Subscripts

0 = entry into the atmosphere;

1 = exit from the atmosphere;

00 = exit from the initial orbit;

$11=$ entry into the final orbit.

Superscripts

- = derivative with respect to dimensionless time;

$\sim \quad$ = variable computed in an inertial system.

\section{Acronyms}

AOT = aeroassisted orbital transfer;

GEO = geosynchronous Earth orbit;

HEO = high Earth orbit;

LEO = low Earth orbit;

SGRA = sequential gradient-restoration algorithm;

TPBVP = two-point boundary-value problem. 


\section{Introduction}

Saving propellant weight and increasing the payload are among the most important problems of space transportation. Orbital transfer from a high Earth orbit (HEO) to a low Earth orbit (LEO) can be made more economic if the aeroassisted orbital transfer (AOT) mode is employed. In particular, HEO can be a geosynchronous Earth orbit (GEO).

In the AOT mode, use is made of the aerodynamic forces in order to achieve the proper amount of velocity depletion during the atmospheric pass. Here, the intent is to achieve a specified apogee following the atmospheric exit, while minimizing the overall propellant consumption and keeping the peak heating rate within reasonable bounds during the atmospheric pass.

Aeroassisted orbital transfer is not only important for HEO-to-LEO transfer maneuvers, but may prove to be indispensable to future planetary flights. In particular, this statement refers to lunar return vehicles, Mars exploration vehicles, and Mars return vehicles (Refs. 1-3). Indeed it is known that, for a round-trip Earth-to-Mars mission, the total characteristic velocity of the AOT mode is about half that of the all-propulsive mode.

While the AOT prospects are clearly bright, to take proper advantage of them it is necessary that guidance and control systems be designed with care. In turn, it is imperative that optimal trajectories be studied (Refs. 4-8), since they supply 
the ideal benchmark that guidance trajectories should strive to approach (Refs. 9-14).

In this report, GEO-to-LEO optimal trajectories are studied with the understanding that the atmospheric pass is made with the engine shut off and is controlled via the lift coefficient so as to deplete excess velocity. The entry and exit values of the path inclination are free, and the criterion of optimization is the total characteristic velocity, which is being minimized.

With the above ideas in mind, this paper is organized as follows. Section 2 presents the system description. In section 3 , several optimization problems are formulated; in Section 4, the data used in the numerical experiments on optimal trajectories are presented.

In Sections 5-6, an ideal optimal trajectory is determined analytically for lift coefficient unbounded. This trajectory is called grazing trajectory, because the atmospheric pass is made by flying at constant altitude along the edge of the atmosphere until the excess velocity is depleted. For the grazing trajectory, the lift coefficient varies in such a way that the lift, the centrifugal force due to the Earth's curvature, the weight, and the Coriolis force due to the Earth's rotation are in static balance. Also, the grazing trajectory minimizes the total characteristic velocity and simultaneously nearly minimizes the peak values of the altitude drop, the dynamic pressure, and the heating rate. 
In Sections 7-8, starting from the grazing trajectory results, a real optimal trajectory is determined numerically for lift coefficient bounded from both below and above. This trajectory is characterized by atmospheric penetration with the smallest possible entry angle, followed by flight at the lift coefficient lower bound. Consistently with the grazing trajectory behavior, the real optimal trajectory minimizes the total characteristic velocity and simultaneously nearly minimizes the peak values of the altitude drop, the dynamic pressure, and the heating rate. Finally, the conclusions are given in Section 9 .

These results have important implications for the design of guidance and control systems. Indeed, it appears possible to develop a nominal trajectory having a nearly-constant lift coefficient and performance close to that of the optimal trajectory; this concept is of particular interest for low 1ift-to-drag ratio spacecraft with a narrow lift range (Refs.11-12). Also, it appears that the properties of minimum characteristic velocity, minimum peak altitude drop, minimum peak dynamic pressure, and minimum peak heating rate are achieved via the same control distribution. Hence, one surmises that AOT guidance and control systems can be designed by concentrating on the peak altitude drop, because all the other performance indexes are directly connected with this quantity. 
2. System Description

We consider coplanar, aeroassisted orbital transfer from a high Earth orbit (HEO) to a low Earth orbit (LEO). In particular, HEO can be a geosynchronous Earth orbit (GEO). We employ the following assumptions: (i) the initial and final orbits are circular and equatorial; (ii) two impulses are employed, one at the exit from the initial orbit and one at the entry into the final orbit; (iii) the gravitational field is central and is governed by the inverse square law. The four key points of the maneuver are these: point 00, exit from the initial orbit; point 0 , entry into the atmosphere; point 1, exit from the atmosphere; and point 11, entry into the final orbit. See Fig. 1.

The maneuver starts at point 00 in high Earth orbit with a tangential propulsive burn having characteristic velocity $\Delta \mathrm{V}_{00}$; here, the spacecraft enters an elliptical transfer orbit, connecting the points 00 and 0 ; this elliptical transfer orbit is such that its apogee occurs at $r_{00}$. At point 0 , the spacecraft enters the atmosphere; after traversing the upper layers of the atmosphere, it exits the atmosphere at point 1; during the atmospheric pass, the velocity of the spacecraft is depleted, due to the aerodynamic drag. At point 1 , the spacecraft enters an elliptical transfer orbit connecting the points 1 and 11 ; this elliptical transfer orbit is such that its apogee occurs at $\mathrm{r}_{11}$. The maneuver ends at point 11 with a tangential propulsive burn having characteristic velocity $\Delta \mathrm{V}_{11}$; here, the spacecraft enters the low Earth orbit, in that the magnitude of $\Delta \mathrm{V}_{11}$ is such that the desired circularization into LEO is achieved. 
For the atmospheric portion $\left(h \leq h_{a}\right)$ of the trajectory of the AOT spacecraft, we employ an Earth-fixed system; for the space portion of the trajectory $\left(h>h_{a}\right)$, we employ an inertial system. For $h \leq h_{a}$, we compute the air density using the US Standard Atmosphere, 1976 (Ref. 15); for $h>h_{a}$, we assume that the air density is zero.

2.1. Atmospheric Pass. Because the initial and final orbits are equatorial, the atmospheric portion of the trajectory of the AOT vehicle is also equatorial if the bank angle is zero. We employ the following hypotheses: (i) the atmospheric pass is made by flying eastward; (ii) the engine is shut off; hence, the AOT vehicle behaves as a particle of constant mass; (iii) Coriolis acceleration terms generated by the Earth's rotation are considered, while transport acceleration terms are neglected; (iv) the spacecraft is controlled via the lift coefficient; (v) under extreme hypersonic conditions, the dependence of the aerodynamic coefficients on the Mach number and the Reynolds number is disregarded.

2.2. Differential system. With the above assumptions, and upon normalizing the flight time to unity, the equations of motion in an Earth-fixed system are given by

$$
\begin{aligned}
& \dot{\mathrm{h}}=\tau[\mathrm{V} \sin \gamma], \\
& \dot{\mathrm{V}}=\tau[-\mathrm{D} / \mathrm{m}-\mathrm{g} \sin \gamma], \\
& \dot{\gamma}=\tau[\mathrm{L} / \mathrm{mV}+(\mathrm{V} / \mathrm{r}-\mathrm{g} / \mathrm{V}) \cos \gamma+2 \omega],
\end{aligned}
$$

with $0 \leq t \leq 1$. In the above equations, 


$$
r=r_{e}+h, \quad g=\mu / r^{2}=\mu /\left(r_{e}+h\right)^{2},
$$

where $\mu$ denotes the Earth's gravitational constant. In addition, the aerodynamic forces are given by

$$
D=(1 / 2) C_{D} \rho S V^{2}, \quad L=(1 / 2) C_{L} \rho S V^{2}
$$

with $\rho=\rho(h)$. In particular, if a quadratic polar is postulated, the relation between the drag coefficient and the lift coefficient is given by

$$
C_{D}=K_{0}+K_{1} C_{L}+K_{2} C_{L}^{2}
$$

2.3. Control Constraint. To obtain realistic solutions, the presence of upper and lower bounds on the lift coefficient is necessary. Therefore, the two-sided inequality constraint

$$
C_{L I} \leq C_{L} \leq C_{L U^{\prime}} \quad 0 \leq t \leq 1,
$$

must be satisfied everywhere along the interval of integration. 2.4. Transformation Relations. The following equations allow one to pass from quantities computed in an Earthfixed system to quantities computed in an inertial system, and viceversa:

$$
\begin{aligned}
& \tilde{h}=h, \\
& \tilde{v} \cos \tilde{\gamma}=v \cos \gamma+w r, \\
& \tilde{v} \sin \tilde{\gamma}=v \sin \gamma .
\end{aligned}
$$


While the above equations are nonlinear, they can be solved explicitly to yield inertial quantities in terms of Earth-fixed quantities, and viceversa. One obtains the direct relations

$$
\begin{aligned}
& \tilde{h}=h, \\
& \tilde{v}=\sqrt{\left(V^{2}+2 \omega r V \cos \gamma+\omega^{2} r^{2}\right),} \\
& \tilde{\gamma}=\arctan [V \sin \gamma /(V \cos \gamma+\omega r)],
\end{aligned}
$$

and the inverse relations

$$
\begin{aligned}
& \mathrm{h}=\tilde{\mathrm{h}} \\
& \left.\mathrm{V}=\sqrt{\mathrm{l}} \tilde{\mathrm{V}}^{2}-2 \omega \mathrm{r} \tilde{\mathrm{V}} \cos \tilde{\gamma}+\omega^{2} r^{2}\right), \\
& \gamma=\arctan [\tilde{\mathrm{V}} \sin \tilde{\gamma} /(\tilde{\mathrm{V}} \cos \tilde{\gamma}-\omega r)] .
\end{aligned}
$$

2.5. Boundary Conditions. At the entry into the atmosphere $(t=0)$ and at the exit from the atmosphere $(t=1)$, certain static and dynamic boundary conditions must be satisfied. Specifically, at atmospheric entry, we have

$$
\begin{aligned}
& h_{0}=h_{a}, \\
& r_{00}{ }^{2}\left(2 v_{\star}{ }^{2}-\tilde{v}_{0}^{2}\right)-2 r_{00} r_{a} v^{2}+r_{a}{ }^{2} \tilde{v}_{0}{ }^{2} \cos ^{2} \tilde{\gamma}_{0}=0,
\end{aligned}
$$

where $h_{a}$ is the thickness of the atmosphere and $v_{\star}=\tilde{v}_{a}=\sqrt{ }\left(\mu / r_{a}\right)=$ $7.832 \mathrm{~km} / \mathrm{sec}$ is a reference velocity, i.e., the circular velocity at $r=r_{a}$. In addition, at atmospheric exit, we have

$$
\begin{aligned}
& \mathrm{h}_{1}=\mathrm{h}_{\mathrm{a}^{\prime}} \\
& \mathrm{r}_{11}{ }^{2}\left(2 \mathrm{v}_{*}{ }^{2}-\tilde{\mathrm{v}}_{1}{ }^{2}\right)-2 \mathrm{r}_{11} \mathrm{r}_{\mathrm{a}} \mathrm{v}^{2}+\mathrm{r}^{2}{ }^{2} \tilde{\mathrm{v}}_{1}{ }^{2} \cos ^{2} \tilde{\gamma}_{1}=0 .
\end{aligned}
$$


2.6. Summary. The relations governing the atmospheric pass include the differential system (1)-(3), the control constraint (4), and the boundary conditions $(8)-(9)$, in which the inertial quantities are related to the Earth-fixed quantities via the transformation relations (6). In this formulation, the independent variable is the time $t, 0 \leq t \leq 1$. The dependent variables include the state variables $h(t), V(t), \gamma(t)$, the control variable $C_{L}(t)$, and the parameter $\tau$. 
3. Optimal Control Problems

Subject to the previous constraints, different optimization problems can be formulated, depending on the performance index chosen. The resulting optimal control problems are either of the Bolza type or the Chebyshev type.

Problem (P1). Minimum Energy. It is required to minimize the energy necessary for orbital transfer. A measure of this energy is the total characteristic velocity $\Delta \tilde{V}$, the sum of the initial characteristic velocity $\Delta \tilde{\mathrm{V}}_{00}$, associated with the propulsive burn from HEO, and the final characteristic velocity $\Delta \tilde{V}_{11}$, associated with the propulsive burn into LEO. Clearly,

$$
\text { II }=\Delta \tilde{V}=\Delta \tilde{V}_{00}+\Delta \tilde{V}_{1 I^{\prime}}
$$

with

$$
\begin{aligned}
& \Delta \tilde{\mathrm{v}}_{00}=V\left(\mathrm{r}_{\mathrm{a}} / \mathrm{r}_{00}\right) \mathrm{v}_{\star}-\left(\mathrm{r}_{\mathrm{a}} / \mathrm{r}_{00}\right) \tilde{\mathrm{v}}_{0} \cos \tilde{\gamma}_{0}, \\
& \Delta \tilde{\mathrm{v}}_{11}=r\left(\mathrm{r}_{\mathrm{a}} / \mathrm{r}_{11}\right) \mathrm{v}_{*}-\left(\mathrm{r}_{\mathrm{a}} / \mathrm{r}_{11}\right) \tilde{\mathrm{v}}_{1} \cos \tilde{\gamma}_{1} .
\end{aligned}
$$

Here, the inertial quantities are related to the Earth-fixed quantities via the transformation relations (6).

Problem (P2). Minimum Peak Altitude Drop. It is required to minimize the peak value of the altitude drop. The performance index is given by

$$
\text { I2 } \max _{t}\left(h_{a}-h\right), \quad 0 \leq t \leq 1 .
$$

Here, $h_{a}=120 \mathrm{~km}$ denotes the altitude corresponding to the outer edge of the atmosphere. 
Problem (P3). Minimum Peak Dynamic Pressure. It is required to minimize the peak value of the dynamic pressure. The performance index is given by

$$
\begin{array}{ll}
I 3=\max _{t}(D P), & 0 \leq t \leq 1, \\
D P=(1 / 2) \rho V^{2} . &
\end{array}
$$

Problem (P4). Minimum Peak Heating Rate. It is required to minimize the peak value of the heating rate. The performance index is given by

$$
\begin{aligned}
I 4 & =\max _{t}(H R), & 0 \leq t \leq 1, \\
H R & =C \sqrt{\left(\rho / \rho_{*}\right)\left(V / V_{\star}\right)^{3.07} .} &
\end{aligned}
$$

Here, $\rho_{*}=0.3097 \mathrm{E}-03 \mathrm{~kg} / \mathrm{m}^{3}$ is a reference density (density at the reference altitude $\left.\mathrm{h}_{*}=\mathrm{h}_{\mathrm{a}} / 2=60 \mathrm{~km}\right), \mathrm{v}_{*}=\tilde{\mathrm{V}}_{\mathrm{a}}=7.832 \mathrm{~km} / \mathrm{sec}$ is a reference velocity, and the constant $C=282.3 \mathrm{~W} / \mathrm{cm}^{2}$ represents the stagnation point heating rate at $\rho=\rho_{*}$ and $V=V_{*}$, based on a nose radius of one foot. 
4. Experimental Data

The following data are used in the numerical experiments on optimal trajectories.

Physical Constants. The physical constants used in the computations are as follows: the radius of the Earth is $\mathrm{r}_{\mathrm{e}}=6378 \mathrm{~km}$; the radius of the outer edge of the atmosphere is $\mathrm{r}_{\mathrm{a}}=6498 \mathrm{~km}$; the thickness of the atmosphere is $h_{a}=120 \mathrm{~km}$; the Earth's gravitational constant is $\mu=0.3986 \mathrm{E}+06 \mathrm{~km}^{3} / \mathrm{sec}^{2}$; the Earth's angular velocity is $\omega=0.7292 \mathrm{E}-04 \mathrm{rad} / \mathrm{sec}$.

Atmospheric Model. The atmospheric model used is the US Standard Atmosphere, 1976 (Ref. 15). In this model, the values of the density are tabulated at discrete altitudes. For intermediate altitudes, the density is computed by assuming an exponential fit for the function $\rho(h)$.

Transfer Maneuver. A GEO-to-LEO transfer maneuver is considered. The GEO radius is $r_{00}=42164 \mathrm{~km}$, corresponding to $r_{00} / r_{a}=6.4888$. The LEO radius is $r_{11}=6708 \mathrm{~km}$, corresponding to $r_{11} / r_{a}=1.0323$. Spacecraft S1. See Refs. 13-14. The mass per unit reference surface area is $\mathrm{m} / \mathrm{S}=300 \mathrm{~kg} / \mathrm{m}^{2}$. The arag polar $C_{D}\left(C_{L}\right)$ is shown in Fig. 2A and is represented by Eq. (3b), with $\mathrm{K}_{0}=0.10$, $\mathrm{K}_{1}=0.00, \mathrm{~K}_{2}=1.11$. The lift coefficient is subject to Ineq. (4), with $C_{L L}=-0.90$ and $C_{L U}=+0.90$. The maximum lift-to-drag ratio modulus is $E_{\max }=1.50$ and occurs for $C_{L}=0.30$.

In the numerical experiments, the possibility of a smaller lift range is considered via three alternative values for the lift coefficient lower bound: 
(i) $C_{L L}=-0.90$, (ii) $C_{L L}=-0.70$, (iii) $C_{L L}=-0.50$.

For these cases, the maximum lift-to-drag ratio modulus is $\mathrm{E}_{\max }=1.50$ and occurs for $C_{L}=0.30$.

Spacecraft S2. See Ref. 11. The mass per unit reference surface area is $\mathrm{m} / \mathrm{S}=117.3 \mathrm{~kg} / \mathrm{m}^{2}$. The drag polar $C_{D}\left(C_{L}\right)$ is shown in Fig. $2 \mathrm{~B}$ and is represented by Eq. (3b), with $\mathrm{K}_{0}=1.235$, $\mathrm{K}_{1}=-2.379, \mathrm{~K}_{2}=-5.473$. The $1 \mathrm{ift}$ coefficient is subject to Ineq. (4), with $C_{L L}=-0.47$ and $C_{L U}=-0.21$. The maximum lift-todrag ratio modulus is $E_{\max }=0.42$ and occurs for $C_{L}=-0.47$. In the numerical experiments, the possibility of a smaller lift range is considered via three alternative values for the lift coefficient lower bound:
(i) $C_{L L}=-0.47$,
(ii) $C_{\text {LL }}=-0.38$,
(iii) $C_{L L}=-0.27$

For these cases, the values of the maximum lift-to-drag ratio modulus are

$$
\text { (i) } \mathrm{E}_{\max }=0.42 \text {, (ii) } \mathrm{E}_{\max }=0.28 \text {, (iii) } \mathrm{E}_{\max }=0.18
$$

and occur for $C_{L}=C_{L L}$.

It must be noted that, for spacecraft 52 , the lift coefficient $C_{L}=-0.47$ corresponds to the angle of attack $\alpha=27 \mathrm{deg}$; $C_{L}=-0.38$ corresponds to $\alpha=17$ deg; $C_{L}=-0.27$ corresponds to $\alpha=10 \mathrm{deg}$; and $C_{L}=-0.21$ corresponds to $\alpha=7 \mathrm{deg}$ (see Ref. 11). 
5. Ideal Optimal Trajectory, Lift Coefficient Unbounded An ideal optimal trajectory can be determined analytically if one minimizes the total characteristic velocity (10), subject to all of the constraints of section 2, except the control constraint (4). This can be done via the four-step procedure described below. Step 1. Ignore not only the control constraint (4), but also the differential equations (1) and the static boundary conditions ( $8 a)$ and (9a). Then, consider the problem of minimizing the total characteristic velocity (10) with respect to the terminal state components $\tilde{\mathrm{V}}_{0}, \tilde{\gamma}_{0}, \tilde{\mathrm{V}}_{1}, \tilde{\gamma}_{1}$ which satisfy the dynamic boundary conditions ( $8 \mathrm{~b})$ and (9b). Clearly, this is a mathematical programming problem in which the initial state components $\tilde{v}_{0}, \tilde{\gamma}_{0}$ and the final state components $\tilde{v}_{1}, \tilde{\gamma}_{1}$ appear separately in both the objective function and the constraining relations. Therefore, the solution can be obtained by solving two separate mathematical programming problems: (i) the minimization of the HEO characteristic velocity $(10 \mathrm{~b})$ with respect to the initial state components $\tilde{v}_{0}, \tilde{\gamma}_{0}$ which satisfy the constraint ( $8 \mathrm{~b})$; and (ii) the minimization of the LEO characteristic velocity (10c) with respect to the final state components $\tilde{V}_{1}, \tilde{\gamma}_{1}$ which satisfy the constraint $(9 \mathrm{~b})$. This yields the inertial solutions

$$
\begin{aligned}
& \tilde{v}_{0}=v_{\star} \sqrt{ }\left[2 r_{00} /\left(r_{00}+r_{a}\right)\right], \\
& \tilde{v}_{1}=v_{\star} \sqrt{ }\left[2 r_{11} /\left(r_{11}+r_{a}\right)\right],
\end{aligned}
$$

and 


$$
\begin{aligned}
& \tilde{\gamma}_{0}=0, \\
& \tilde{\gamma}_{1}=0 .
\end{aligned}
$$

In turn, because of the inverse transformation relations (7), the inertial solutions (17)-(18) imply the fixed-Earth solutions

$$
\begin{aligned}
& v_{0}=v_{\star} /\left[2 r_{00} /\left(r_{00}+r_{a}\right)\right]-w r_{a^{\prime}} \\
& v_{1}=v_{\star} /\left[2 r_{11} /\left(r_{11}+r_{a}\right)\right]-w r_{a^{\prime}}
\end{aligned}
$$

and

$$
\begin{aligned}
& \gamma_{0}=0, \\
& \gamma_{1}=0 .
\end{aligned}
$$

Step 2. Because of the result (20), it is natural to postulate the following altitude/path inclination solutions

$$
\begin{array}{ll}
h(t)=h_{a^{\prime}} & 0 \leq t \leq 1, \\
\gamma(t)=0, & 0 \leq t \leq 1,
\end{array}
$$

which are clearly consistent with the differential equation (la) plus the static boundary conditions (8a) and (9a).

Step 3. After observing that, because of (21b),

$$
\dot{\gamma}(t)=0, \quad 0 \leq t \leq 1,
$$

we see that the differential equation (1c) reduces to

$$
L=-m\left(V^{2} / r_{a}-g_{a}\right)-m(2 \omega V)
$$


which yields the required lift coefficient

$$
C_{L}=-C_{1}\left[1-\left(V_{\star} / V\right)^{2}\right]-C_{2}\left(V_{\star} / V\right)
$$

where the dimensionless constants $C_{1}, C_{2}$ are given by

$$
C_{1}=2 m / \rho_{a} S r_{a}, \quad C_{2}=4 m \omega / \rho_{a} S v_{\star} .
$$

In particular, the entry and exit values of the required lift coefficient are given by the relations

$$
\begin{aligned}
& C_{L 0}=-C_{1}\left[1-\left(v_{\star} / V_{0}\right)^{2}\right]-C_{2}\left(v_{\star} / V_{0}\right), \\
& C_{L I}=-C_{1}\left[1-\left(v_{\star} / V_{1}\right)^{2}\right]-C_{2}\left(v_{*} / V_{1}\right),
\end{aligned}
$$

which must be employed in conjunction with Eqs. (19).

Step 4. It remains to verify whether the differential equation (1b) can be satisfied. Simple manipulations, omitted for the sake of brevity, show that this is precisely the case if the velocity-dimensionless time relation $V(t)$ is determined through the inverse relation $t(V)$ represented by

$$
t=\left(c_{3} / \tau\right) \int_{V}^{V_{0}}\left(v_{\star} / C_{D} v^{2}\right) d v
$$

Here, the dimensional constant $c_{3}$ (sec) and the flight time $\tau$ (sec) are given by

$$
\begin{aligned}
& \mathrm{C}_{3}=2 \mathrm{~m} / \rho_{\mathrm{a}} \mathrm{SV}_{*} \text {, } \\
& \tau=c_{3} \int_{V_{1}}^{V_{0}}\left(v_{*} / C_{D} v^{2}\right) d v \text {, }
\end{aligned}
$$

and the drag coefficient $C_{D}$ is given by Eq. (3b) employed in conjunction with Eqs. (24). 
6. Properties of the Ideal Optimal Trajectory

The ideal optimal trajectory determined for lift coefficient unbounded is governed by Eqs.(17)-(26) and satisfies all of the constraints of section 2 except the control inequality constraint (4). This trajectory is called grazing trajectory because the atmospheric pass is made by flying at constant altitude along the edge of the atmosphere. Thus, the complete transfer maneuver includes: (i) a descending flight branch connecting an apogee located at GEO to a perigee located on the atmospheric edge; (ii) a level flight branch flown along the atmospheric edge; and (iii) an ascending flight branch connecting a perigee located on the atmospheric edge to an apogee located at LEO.

6.1. Characteristic Velocity. By combining Eqs. (10) and (17)-(18), we see that the characteristic velocity of the grazing trajectory is given by

$$
\begin{aligned}
& I I=\Delta \tilde{V}=\Delta \tilde{V}_{00}+\Delta \tilde{V}_{1 I}, \\
& \left.\Delta \tilde{v}_{00}=v_{\star} \sqrt{(} r_{a} / r_{00}\right)-v_{\star}\left(r_{a} / r_{00}\right) v\left[2 r_{00} /\left(r_{00}+r_{a}\right)\right], \\
& \Delta \tilde{v}_{11}=v_{*} /\left(r_{a} / r_{11}\right)-v_{*}\left(r_{a} / r_{11}\right) \sqrt[V]{ }\left[r_{11} /\left(r_{11}+r_{a}\right)\right] .
\end{aligned}
$$

For comparison purposes, consider the Hohmann transfer maneuver, which is flown totally in space by navigating in the region bounded by $r=r_{00}$ and $r=r_{11}$. For this trajectory, the characteristic velocity is given by

$$
\begin{aligned}
& \text { II }=\Delta \tilde{V}=\Delta \tilde{V}_{00}+\Delta \tilde{V}_{11}, \\
& \left.\Delta \tilde{V}_{00}=v_{*} V\left(r_{a} / r_{00}\right)-v_{*} \Omega\left(r_{a} / r_{00}\right) \Omega 2 r_{11} /\left(r_{00}+r_{11}\right)\right],
\end{aligned}
$$




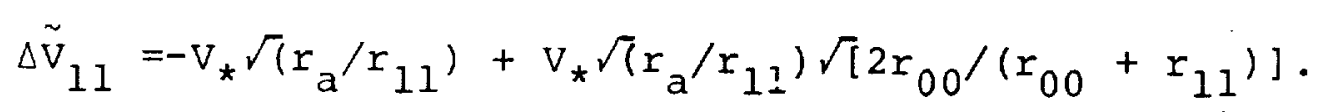

Table 1 compares the grazing solution (first line) and the Hohmann transfer solution (third line) and shows that the total characteristic velocity of the grazing solution is less than $40 \%$ of that of the Hohmann transfer solution. This illustrates the savings in propellant mass which are possible by employing aeroassisted orbital transfer techniques instead of Hohmann transfer techniques.

6.2. Peak Altitude Drop. For the grazing trajectory, the functional (11) takes the value

$$
I 2=\max _{t}\left(h_{a}-h\right)=0 .
$$

Therefore, the grazing trajectory simultaneously minimizes the peak value of the altitude drop.

6.3. Peak Dynamic Pressure.For the grazing trajectory, the functional (12) takes the value

$$
I 3=\max _{t}(D P)=(1 / 2) \rho_{a} V_{0}^{2},
$$

meaning that the peak dynamic pressure occurs at atmospheric entry $(t=0)$. Note that the performance index (12) is dominated by the density rather than the velocity in the following sense: during the atmospheric penetration, the density variations are much larger than the velocity variations. Hence, it is felt plausible that the grazing trajectory nearly minimizes the functional (12).

6.4. Peak Heating Rate. For the grazing trajectory, the functional (13) takes the value 


$$
I 4=\max _{t}(H R)=C V\left(\rho_{a} / \rho_{\star}\right)\left(V_{0} / V_{*}\right)^{3.07},
$$

meaning that the peak heating rate occurs at atmospheric entry $(t=0)$. Once more, because the performance index (13) is dominated by the density rather than the velocity, it is felt plausible that the grazing trajectory nearly minimizes the functional (13).

6.5. Implications. Associated with the grazing trajectory $\left(C_{L}\right.$ unbounded) are the following engineering implications for a real optimal trajectory $\left(\mathrm{C}_{\mathrm{L}}\right.$ bounded).

(i) The characteristic velocity of the grazing trajectory (27) constitutes a lower bound for the characteristic velocity of a real optimal trajectory. Therefore, it constitutes a benchmark that one should strive to approach with a real optimal trajectory.

(ii) Because the grazing trajectory simultaneously minimizes or nearly minimizes the performance indexes (II) through (I4), one surmises that the same general behavior might hold for a real optimal trajectory.

(iii) Along a grazing trajectory, the lift, the centrifugal force due to the Earth's curvature, the weight, and the coriolis force due to the Earth's rotation are in static equilibrium.

(iv) For a grazing trajectory, the velocity decreases monotonically between the entry value (19a) and the exit value (19b), and the required lift coefficient increases monotonically between the entry value (25a) and the exit value (25b). For the 
two spacecraft under consideration, the terminal values of $C_{L}$ are given by

$$
\begin{aligned}
& \text { (S1) } \quad C_{L 0}=-1921.3, \quad C_{L 1}=-56.6, \\
& \text { (S2) } \quad C_{L 0}=-750.6, \quad C_{L 1}=-22.1,
\end{aligned}
$$

with the following meaning: not only is the required lift coefficient negative everywhere, but it undershoots by a considerable amount the lower bound $C_{L L}$. This means that the grazing trajectory is only an ideal trajectory, hence not flyable.

(v) To sum up, while the grazing trajectory was computed neglecting the two-sided inequality constraint (4), the nature of the grazing solution is such that the upper bound inequality $C_{L} \leq C_{L U}$ is satisfied everywhere, while the lower bound inequality $C_{L} \geq C_{L L}$ is violated everywhere. Even though the grazing trajectory is not flyable, this suggests the idea that, for a real optimal trajectory, the control distribution might be of the form $C_{L}=C_{L I}$. This idea is explored in the following section. 
7. Real Optimal Trajectory, Lift Coefficient Bounded

A real optimal trajectory can be determined numerically by accounting for all of the constraints of section 2 , including the control constraint (4). Two alternative procedures are now presented.

7.1. Solution via TPBVP. The grazing trajectory of sections 5-6 satisfies all of the constraints of section 2, except the two-sided inequality constraint (4). Specifically, the lower bound inequality $C_{I} \geq C_{L I}$ is violated everywhere. This suggests the idea that the control distribution of the real optimal trajectory might be of the form $C_{L}=C_{L I}$.

If the hypothesis $C_{L}=C_{L L}$ is made, the resulting trajectory is described by the differential system

$$
\begin{aligned}
& \dot{\mathrm{h}}=\tau[\mathrm{Vsin} \gamma], \\
& \dot{\mathrm{V}}=\tau\left[-\mathrm{D}\left(\mathrm{h}, \mathrm{V}, \mathrm{C}_{L L}\right) / \mathrm{m}-g \sin \gamma\right], \\
& \dot{\gamma}=\tau\left[\mathrm{L}\left(\mathrm{h}, \mathrm{V}, \mathrm{C}_{L L}\right) / \mathrm{mV}+(\mathrm{V} / \mathrm{r}-\mathrm{g} / \mathrm{V}) \cos \gamma+2 \omega\right], \\
& \dot{\tau}=0,
\end{aligned}
$$

which must be solved in conjunction with the initial conditions

$$
\begin{aligned}
& \mathrm{h}_{0}=\mathrm{h}_{\mathrm{a}} \\
& \left(2 \mathrm{r}_{00}^{2}-2 r_{00} r_{a}\right) \mathrm{v}_{\star}^{2}-\mathrm{r}_{00}^{2}\left(\mathrm{v}_{0}^{2}+2 \omega \mathrm{r}_{\mathrm{a}} \mathrm{v}_{0} \cos \gamma_{0}+\omega^{2} \mathrm{r}_{\mathrm{a}}^{2}\right) \\
& +\mathrm{r}_{\mathrm{a}}^{2}\left(\mathrm{v}_{0} \cos \gamma_{0}+\omega \mathrm{r}_{\mathrm{a}}\right)^{2}=0,
\end{aligned}
$$

and the final conditions 


$$
\begin{aligned}
& \mathrm{h}_{1}=\mathrm{h}_{\mathrm{a}} \\
& \left(2 \mathrm{r}_{11}^{2}-2 \mathrm{r}_{11} \mathrm{r}_{\mathrm{a}}\right) \mathrm{v}_{\star}^{2}-\mathrm{r}_{11}^{2}\left(\mathrm{v}_{1}^{2}+2 \omega \mathrm{r}_{\mathrm{a}} \mathrm{v}_{1} \cos \gamma_{1}+\omega^{2} \mathrm{r}_{\mathrm{a}}^{2}\right) \\
& +\mathrm{r}_{\mathrm{a}}^{2}\left(\mathrm{v}_{1} \cos \gamma_{1}+\omega \mathrm{r}_{\mathrm{a}}\right)^{2}=0 .
\end{aligned}
$$

The following comments are pertinent:

(i) the system (33) is a formal modification of the system (1) after accounting for the hypothesis $C_{L}=C_{L I}$ and the fact that, since $\tau$ is a parameter, it can be represented via the differential equation $\dot{\tau}=0$; this is done only for explanation purposes; (ii) the boundary conditions (34b) and (35b) are a formal modification of the boundary conditions ( $8 b)$ and (9b) after accounting for the transformation relations (5)-(6);

(iii) the problem represented by Eqs. (33)-(35) is a twopoint boundary-value problem (TPBVP) in which the unknowns are the functions $h(t), V(t), \gamma(t), \tau(t)$; since there are four differential equations and four boundary conditions, one surmises that a solution might exist; however, because the TPBVP (33)-(35) is nonlinear, the existence of a solution must be confirmed by numerical tests.

7.2. Solution via SGRA. An alternative to solving the previous TPBVP is to employ the sequential gradient-restoration algorithm (SGRA, Refs. 16-18) in order to minimize the functional (10) with respect to the state variables $h(t), v(t), \gamma(t)$, the control variable $C_{L}(t)$, and the parameter $\tau$ which satisfy the differential system (1)-(3), the control constraint (4), and the boundary conditions (8)-(9) rewritten in the form (34)-(35) after replacement 
of inertial quantities with Earth-fixed quantities. Note that SGRA is an iterative algorithm which produces a sequence of feasible solutions, each characterized by a lower value of the functional (10). While SGRA is available in both primal form and dual form (Refs. 17-18), the primal form is more suitable to the solution of hypervelocity flight problems. Hence, it is employed here.

7.3. Numerical Results. In computing numerical solutions via SGRA, the experimental data of section 4 were employed. Hence, a GEO-to-LEO optimal aeroassisted orbital transfer was determined for both Spacecraft Sl (see Refs. 13-14) and Spacecraft S2 (see Ref. 11). For each spacecraft, three different values were considered for the lift coefficient lower bound, specifically, the values (14) for spacecraft Sl and the values (15) for spacecraft S2. The numerical results are shown in Tables $1-2$ and Figs. 3-4. Table 1 compares the characteristic velocity of the real optimal trajectory (second line) with that of the grazing trajectory (first line) and the Hohmann transfer trajectory (third line). clearly, the characteristic velocity of the real optimal trajectory is within $1 \%$ of that of the grazing trajectory and is considerably below (by almost 60\%) that of the Hohmann transfer trajectory. Table 2 presents summary results for the real optimal trajectory of Spacecraft $\mathrm{Sl}$ and spacecraft $\mathrm{S2}$. In Table 2, the following quantities are shown: the entry values of the state variables; the exit values of the state variables; the minimum altitude, the peak dynamic pressure, the peak heating rate, and the flight time; 
the components of the characteristic velocity and the total characteristic velocity. As $C_{L L}$ increases (namely, as the lift coefficient range $C_{L U}-C_{L L}$ decreases), the real optimal trajectory is characterized by a steeper entry, lower minimum altitude, higher peak dynamic pressure, and higher peak heating rate. However, the total characteristic velocity and its components are almost independent of $\mathrm{C}_{\mathrm{LL}}$, hence almost independent of the lift coefficient range.

Figures 3-4 refer to the real optimal trajectories of spacecraft S1 and Spacecraft S2. The following quantities are shown as functions of the dimensional time $\mathrm{T}$ : the altitude $\mathrm{h}$; the velocity $V$; the path inclination $\gamma$; the lift coefficient $C_{L}$; and the density $\rho$. The results confirm the idea that, for the real optimal trajectory, the lift coefficient is constant and equal to the lower bound. As $C_{L L}$ increases (namely, as the lift coefficient range $C_{L U}-C_{L L}$ decreases), the real optimal trajectory changes; in particular, the minimum altitude changes. However, while the changes in minimum altitude are relatively small, they translate into larger density changes, and hence larger changes in $\max (\mathrm{DP})$ and $\max (\mathrm{HR})$. 
8. Properties of the Real Optimal Trajectory

The real optimal trajectory determined for lift coefficient bounded satisfies all of the constraints of section 2 , including the control inequality constraint (4). This trajectory is called nearly-grazing trajectory, because the atmospheric pass is characterized by atmospheric penetration with the smallest possible entry angle, followed by flight at the lift coefficient lower bound. Thus, the complete transfer maneuver includes: (i) a descending flight branch connecting an apogee located at GEO to the atmospheric entry point; (ii) an atmospheric flight branch including a shallow descent followed by a shallower ascent; and (iii) an ascending flight branch connecting the atmospheric exit point to an apogee located at LEO.

8.1. Characteristic Velocity. If we combine Eqs. (8)-(10) and eliminate the terminal velocities, we see that the characteristic velocity components of the nearly-grazing trajectory can be expressed in terms of the terminal path inclinations as follows:

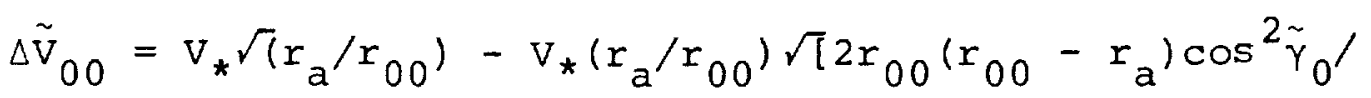

$$
\begin{aligned}
& \left.\left(r_{00}^{2}-r_{a}^{2} \cos ^{2} \tilde{\gamma}_{0}\right)\right]
\end{aligned}
$$

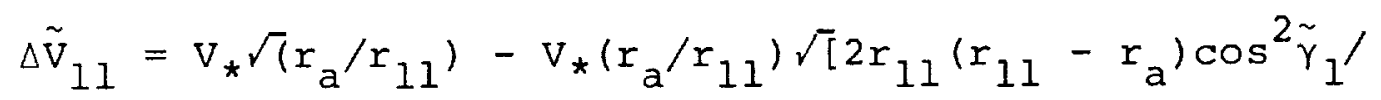

$$
\begin{aligned}
& \left.\left(r_{11}^{2}-r_{a}^{2} \cos ^{2} \tilde{\gamma}_{1}\right)\right]
\end{aligned}
$$

By comparison, we observe that, for the grazing trajectory

$\left(\tilde{\gamma}_{0}=\tilde{\gamma}_{1}=0\right)$, 


$$
\begin{aligned}
& \Delta \tilde{v}_{00 G}=v_{*} \sqrt{\left.\left(r_{a} / r_{00}\right)-v_{*}\left(r_{a} / r_{00}\right) \sqrt{[2} r_{00} /\left(r_{00}+r_{a}\right)\right],}
\end{aligned}
$$

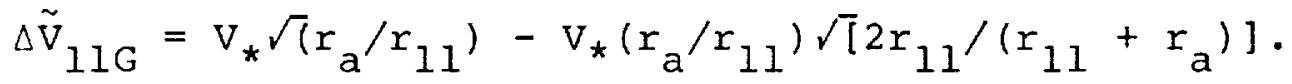

Let the right-hand sides of (36) be expanded in a Taylor series, and let the following approximations be employed:

$$
\begin{aligned}
& \tilde{\gamma}_{0} \cong \gamma_{0}, \\
& \tilde{\gamma}_{1} \cong \gamma_{1} .
\end{aligned}
$$

Then, the following relations can be established between (36) and (37):

$$
\begin{aligned}
& \Delta \tilde{\mathrm{v}}_{00}=\Delta \tilde{\mathrm{v}}_{00 \mathrm{G}}+\mathrm{A}_{0} \gamma_{0}{ }^{2}, \\
& \Delta \tilde{\mathrm{v}}_{11}=\Delta \tilde{\mathrm{V}}_{11 \mathrm{G}}+\mathrm{A}_{1} \gamma_{1}{ }^{2},
\end{aligned}
$$

with

$$
\begin{aligned}
& A_{0}=\left(V_{\star} / \sqrt{2}\right)\left[r_{a} /\left(r_{00}-r_{a}\right)\right]\left[r_{00} /\left(r_{00}+r_{a}\right)\right]^{3 / 2}, \\
& A_{1}=\left(v_{\star} / \sqrt{2}\right)\left[r_{a} /\left(r_{11}-r_{a}\right)\right]\left[r_{11} /\left(r_{11}+r_{a}\right)\right]^{3 / 2} .
\end{aligned}
$$

For the GEO-to-LEO transfer of section 4 , the constants $\mathrm{A}_{0}, \mathrm{~A}_{1}$ take the values

$$
\begin{aligned}
& A_{0}=0.8138 \mathrm{~km} / \mathrm{sec}, \\
& A_{1}=62.03 \mathrm{~km} / \mathrm{sec} .
\end{aligned}
$$

Equations (39) show that the characteristic velocity components of a nearly-grazing trajectory can be contained by making the entry and exit path inclinations as small as possible. For tangential entry 
and exit $\left(\gamma_{0}=\gamma_{1}=0\right)$, the characteristic velocity components of the nearly-grazing trajectory reduce to those of the grazing trajectory. For nontangential entry and exit $\left(\gamma_{0} \neq 0, \gamma_{1} \neq 0\right)$, the characteristic velocity components of the nearly-grazing trajectory are always larger than those of the grazing trajectory. However, the relative differences are small, of order less than $1 \%$ for the examples of Tables 1-2.

8.2. Terminal Velocities. Equations ( $8 b$ ) and (9b) can be solved to obtain the terminal velocities of the nearly-grazing trajectory in terms of the terminal path inclinations as follows:

$$
\begin{aligned}
& \left.\tilde{v}_{0}=v_{*} \sqrt{\left[2 r_{00}\right.}\left(r_{00}-r_{a}\right) /\left(r_{00}^{2}-r_{a}{ }^{2} \cos ^{2} \tilde{\gamma}_{0}\right)\right], \\
& \left.\tilde{v}_{I}=v_{\star} \sqrt{\left[2 r_{11}\right.}\left(r_{11}-r_{a}\right) /\left(r_{11}^{2}-r_{a}{ }^{2} \cos ^{2} \tilde{\gamma}_{1}\right)\right] .
\end{aligned}
$$

By comparison, we observe that, for the grazing trajectory

$$
\begin{aligned}
& \left(\tilde{\gamma}_{0}=\tilde{\gamma}_{1}=0\right) \text {, } \\
& \left.\tilde{v}_{0 G}=v_{*} \sqrt{\left[2 r_{00}\right.} /\left(r_{00}+r_{a}\right)\right], \\
& \tilde{v}_{1 G}=v_{*} \sqrt{\left[2 r_{11} /\left(r_{11}+r_{a}\right)\right] .}
\end{aligned}
$$

Let the right-hand sides of (42) be expanded in a Taylor series, and let the approximations (38) be employed. Then, the following relations can be established between (42) and (43):

$$
\begin{aligned}
& \tilde{v}_{0}=\tilde{v}_{0 G}-B_{0} \gamma_{0}{ }^{2}, \\
& \tilde{v}_{1}=\tilde{v}_{1 G}-B_{1} \gamma_{1}{ }^{2},
\end{aligned}
$$


which approximately imply that

$$
\begin{aligned}
& \mathrm{v}_{0}=\mathrm{v}_{0 \mathrm{G}}-\mathrm{B}_{0} \gamma_{0}{ }^{2}, \\
& \mathrm{v}_{1}=\mathrm{v}_{1 \mathrm{G}}-\mathrm{B}_{1} \gamma_{1}{ }^{2},
\end{aligned}
$$

with

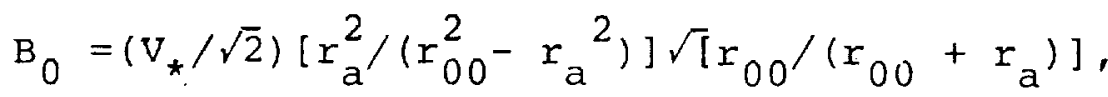

$$
\begin{aligned}
& \left.B_{1}=\left(v_{\star} / \sqrt{2}\right)\left[r_{a}^{2} /\left(r_{11}^{2}-r_{a}^{2}\right)\right] \sqrt{\left[r_{11}\right.} /\left(r_{11}+r_{a}\right)\right] \text {. }
\end{aligned}
$$

For the GEO-to-LEO transfer of section 4 , the constants $\mathrm{B}_{0}, \mathrm{~B}_{1}$ take the values:

$$
\begin{aligned}
& B_{0}=0.1254 \mathrm{~km} / \mathrm{sec}, \\
& B_{1}=60.09 \mathrm{~km} / \mathrm{sec} .
\end{aligned}
$$

Equations (44)-(45) show that the terminal velocities of a nearlygrazing trajectory can be made close to the terminal velocities of a grazing trajectory by making the entry and exit path inclinations as small as possible. For tangential entry and exit $\left(\gamma_{0}=\gamma_{1}=0\right)$, the terminal velocities of the nearly-grazing trajectory reduce to those of a grazing trajectory. For nontangential entry and exit $\left(\gamma_{0} \neq 0, \gamma_{1} \neq 0\right)$, the terminal velocities of the nearly-grazing trajectory are always smaller than those of the grazing trajectory. However, the relative differences are small, of order $10^{-4}$ (entry velocities) or order $10^{-3}$ (exit velocities) for the examples of Tables $1-2$. 
8.3. Perigee Altitude. Let us consider the trajectory described by the spacecraft in the absence of aerodynamic forces and evaluate the energy constant twice, once in terms of the entry conditions and once in terms of the exit conditions. For the nearly-grazing trajectory, this yields the relations

$$
\begin{aligned}
& \mathrm{E}_{0}=\tilde{\mathrm{v}}_{0}^{2} / 2-\mu / \mathrm{r}_{\mathrm{a}}=-\mu /\left(\mathrm{r}_{00}+\mathrm{r}_{\mathrm{P} 0}\right)^{\prime} \\
& \mathrm{E}_{1}=\tilde{\mathrm{v}}_{1}^{2} / 2-\mu / \mathrm{r}_{\mathrm{a}}=-\mu /\left(\mathrm{r}_{11}+\mathrm{r}_{\mathrm{P} 1}\right),
\end{aligned}
$$

in which $\mathrm{r}_{\mathrm{P} 0}, \mathrm{r}_{\mathrm{Pl}}$ are the perigee radii associated with the entry and exit conditions, respectively. The corresponding relations for the grazing trajectory are

$$
\begin{aligned}
& E_{0 G}=\tilde{v}_{0 G}^{2} / 2-\mu / r_{a}=-\mu /\left(r_{00}+r_{a}\right), \\
& E_{1 G}=\tilde{v}_{1 G}^{2} / 2-\mu / r_{a}=-\mu /\left(r_{11}+r_{a}\right) .
\end{aligned}
$$

Upon taking differences, we see that

$$
\begin{aligned}
& \left(\tilde{v}_{0}^{2}-\tilde{v}_{0 G}^{2}\right) / 2=-\mu\left(r_{a}-r_{P 0}\right) /\left(r_{00}+r_{P 0}\right)\left(r_{00}+r_{a}\right), \\
& \left(\tilde{v}_{1}^{2}-\tilde{v}_{1 G}^{2}\right) / 2=-\mu\left(r_{a}-r_{P 1}\right) /\left(r_{11}+r_{P l}\right)\left(r_{11}+r_{a}\right) .
\end{aligned}
$$

Next, we observe that

$$
\begin{aligned}
& r_{a}-r_{P 0}=h_{a}-h_{P 0} \\
& r_{a}-r_{P 1}=h_{a}-h_{P 1}
\end{aligned}
$$

and employ the approximations

$$
\begin{aligned}
& \left(r_{00}+r_{P 0}\right)\left(r_{00}+r_{a}\right) \cong\left(r_{00}+r_{a}\right)^{2}, \\
& \left(r_{11}+r_{P 1}\right)\left(r_{11}+r_{a}\right) \cong\left(r_{11}+r_{a}\right)^{2} .
\end{aligned}
$$


With this understanding, Eqs. (50) can be rewritten as

$$
\begin{aligned}
& \left(\tilde{v}_{0}^{2}-\tilde{v}_{0 G}^{2}\right) / 2=-\mu\left(h_{a}-h_{P 0}\right) /\left(r_{00}+r_{a}\right)^{2}, \\
& \left(\tilde{v}_{1}^{2}-\tilde{v}_{1 G}^{2}\right) / 2=-\mu\left(h_{a}-h_{P 1}\right) /\left(r_{11}+r_{a}\right)^{2} .
\end{aligned}
$$

Because $\mu=r_{a} v_{*}^{2}$, Eqs. (53) imply that

$$
\begin{aligned}
& h_{a}-h_{P 0}=-\left(\tilde{v}_{0}^{2}-\tilde{v}_{0 G}^{2}\right)\left(r_{00}+r_{a}\right)^{2} / 2 r_{a} v_{*}{ }^{2}, \\
& h_{a}-h_{P 1}=-\left(\tilde{v}_{1}^{2}-\tilde{v}_{1 G}^{2}\right)\left(r_{11}+r_{a}\right)^{2} / 2 r_{a} v_{*}^{2} .
\end{aligned}
$$

Note that, in the light of (44),

$$
\begin{aligned}
& \tilde{\mathrm{v}}_{0}^{2}-\tilde{\mathrm{v}}_{0 \mathrm{G}}^{2} \cong-2 \mathrm{~B}_{0} \tilde{\mathrm{v}}_{0 \mathrm{G}^{\gamma_{0}}}{ }^{2}, \\
& \tilde{\mathrm{v}}_{1}^{2}-\tilde{\mathrm{v}}_{1 \mathrm{G}}^{2} \cong-2 \mathrm{~B}_{1} \tilde{\mathrm{v}}_{1 \mathrm{I}_{1}{ }^{2}}{ }^{2} .
\end{aligned}
$$

Hence, the altitude drops of the ideal perigees can be written as

$$
\begin{aligned}
& h_{a}-h_{p 0}=c_{0} \gamma_{0}{ }^{2}, \\
& h_{a}-h_{P I}=c_{1} \gamma_{1}{ }^{2},
\end{aligned}
$$

where

$$
\begin{aligned}
& c_{0}=r_{a} r_{00} /\left(r_{00}-r_{a}\right), \\
& c_{1}=r_{a} r_{11} /\left(r_{11}-r_{a}\right) .
\end{aligned}
$$

For the GEO-to-LEO transfer of section 4 , the constants $C_{0}, C_{1}$ take the values

$$
\mathrm{C}_{0}=7682 \mathrm{~km} \text {, }
$$




$$
C_{1}=207565 \mathrm{~km} \text {. }
$$

Equations (56) show that the altitude drop of the ideal perigee of a nearly-grazing trajectory can be made small by making the entry and exit path inclinations as small as possible. For tangential entry and exit $\left(\gamma_{0}=\gamma_{1}=0\right)$, the altitude arop of the ideal perigee of a nearly-grazing trajectory reduces to that of a grazing trajectory, which is zero. For nontangential entry and exit $\left(\gamma_{0} \neq 0, \gamma_{1} \neq 0\right)$, the altitude drop of the ideal perigee is nonzero. In particular, Eq. (56a) yields a good approximation to the minimum altitude of a nearly-grazing trajectory (within $1 \mathrm{~km}$ ).

8.4. Minimum Altitude. The perigee altitude determined with either of Eqs. (56) constitutes an approximation to $h_{m i n}$, the minimum altitude of the nearly-grazing trajectory. The approximation is more accurate if Eq. (56a) is used instead of Eq. (56b), due to the fact that the descending branch of the nearly-grazing trajectory is shorter in time than the ascending branch. Hence, the effects due to the aerodynamic forces are smaller in the descending branch than in the ascending branch.

Alternatively, one might regard the left-hand sides of Eqs. (56) as approximations to $\max _{t}\left(h_{a}-h\right)$, the maximum altitude drop of the nearly-grazing trajectory. To contain $\max _{t}\left(h_{a}-h\right)$, one must make the right-hand sides of Eqs. (56) as small as possible. Hence, the entry and exit path inclinations should be as small as possible. One surmises that the solution of Problem (P2), minimum peak altitude drop, is nearly the same as the solution of Problem (PI), minimum energy. 
8.5. Dynamic Pressure. During the atmospheric pass of an AOT trajectory, both the density and the velocity change, and hence the dynamic pressure (12b) changes. The ratio of the instantaneous dynamic pressure to the entry dynamic pressure is given by

$$
\mathrm{DP} /(\mathrm{DP})_{0}=\left(\rho / \rho_{\mathrm{a}}\right)\left(\mathrm{V} / \mathrm{V}_{0}\right)^{2}
$$

While the velocity ratio $\mathrm{V} / \mathrm{V}_{0}$ is of $O(1)$, the density ratio $\mathrm{p} / \mathrm{\rho}$ is of $O\left(10^{3}\right)$ to $O\left(10^{4}\right)$, depending on the particular trajectory. Therefore, to contain the dynamic pressure, one must act primarily on the density, hence the altitude, hence the drop of altitude. One surmises that the solution of Problem (P3), minimum peak dynamic pressure, is nearly the same as the solution of Problem (P2), minimum peak altitude drop; hence, it is nearly the same as the solution of Problem (PI), minimum energy.

8.6. Heating Rate. During the atmospheric pass of an AOT trajectory, both the density and the velocity change, and hence the heating rate ( $13 \mathrm{~b})$ changes. The ratio of the instantaneous heating rate to the entry heating rate is given by

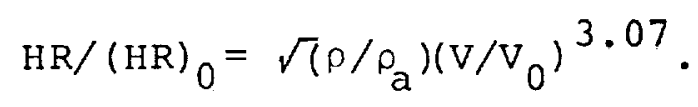

While the velocity ratio $\mathrm{V} / \mathrm{v}_{0}$ is of $O(1)$, the density ratio $\rho / \rho_{a}$ is of $O\left(10^{3}\right)$ to $O\left(10^{4}\right)$, depending on the particular trajectory. Therefore, to contain the heating rate, one must act primarily on the density, hence the altitude, hence the drop of altitude. One surmises that the solution of Problem (P4), minimum peak heating rate, is nearly the same as the solution of : 
Problem (P2), minimum peak altitude drop; hence, it is nearly the same as the solution of Problem (PI), minimum energy. 8.7. Lift Coefficient. For a nearly-grazing trajectory, both $\gamma$ and $\dot{\gamma}$ are small along a major portion of the trajectory; in particular, they are small in the region past the minimum altitude point. For $\gamma$ and $\dot{\gamma}$ small, Eq. (Ic) can be rewritten as

$$
L=(I / 2) C_{L} \rho S V^{2} \cong-m\left(V^{2} / x-g\right)-m(2 \omega V)
$$

Because the atmospheric pass is executed at supercircular velocities, the centrifugal force due to the Earth's curvature $m\left(v^{2} / r\right)$ always exceeds the weight $\mathrm{mg}$; also, the Coriolis force $\mathrm{m}(2 \omega \mathrm{V})$ is directed upward. Because both terms on the right-hand side of (61) are negative, the required lift is negative; hence, the required lift coefficient is negative. To maximize the instantaneous flight altitude, the density $\rho$ should be as small as possible; hence, the lift coefficient $C_{L}$ should be as negative as possible. To sum up, the control $C_{L}=C_{L L}$ allows the flight altitude to be as high as possible, and this is why the solutions of Problems (PI) through (P4) are nearly the same.

8.8. Summary. The real optimal trajectory determined in the presence of the control constraint (4) is a nearly-grazing trajectory and is flown with the lift coefficient at the lower bound, $C_{L}=C_{L L}$. The control distribution $C_{L}=C_{L L}$ not only minimizes the characteristic velocity of Problem (PI), but simultaneously nearly minimizes the peak altitude drop of Problem (P2), the peak dynamic pressure of Problem (P3), and the peak heating rate of Problem (P4). 
9. Conclusions

This paper is concerned with the optimization of trajectories for coplanar, aeroassisted orbital transfer (AOT) from a geosynchronous Earth orbit (GEO) to a low Earth orbit (LEO). It is assumed that the initial and final orbits are circular, that the gravitational field is central and is governed by the inverse square law, and that two impulses are employed, one at GEO exit and one at LEO entry. During the atmospheric pass, the trajectory is controlled via the lift coefficient in such a way that the total characteristic velocity is minimized. First, an ideal optimal trajectory is determined analytically for lift coefficient unbounded. This trajectory is called grazing trajectory, because the atmospheric pass is made by flying at constant altitude along the edge of the atmosphere until the excess velocity is depleted. For the grazing trajectory, the lift coefficient varies in such a way that the lift, the centrifugal force due to the Earth's curvature, the weight, and the Coriolis force due to the Earth's rotation are in static balance. Also, the grazing trajectory minimizes the total characteristic velocity and simultaneously nearly minimizes the peak values of the altitude drop, the dynamic pressure, and the heating rate.

Next, starting from the grazing trajectory results, a real optimal trajectory is determined numerically for lift coefficient bounded from both below and above. This trajectory is characterized by atmospheric penetration with the smallest possible entry angle, followed by flight at the lift coefficient 
lower bound. It is called nearly-grazing trajectory, in that its control distribution is the closest feasible control to that of the grazing trajectory. Consistently with the grazing trajectory behavior, the nearly-grazing trajectory minimizes the total characteristic velocity and simultaneously nearly minimizes the peak values of the altitude drop, the dynamic pressure, and the heating rate. 


\section{References}

1. BOSTON, P. J., Editor, The Case for Mars, Univelt, San Diego, California, 1984.

2. REIBER, D. B., Editor, The NASA Mars Conference, Univelt, San Diego, California, 1988.

3. REA, D. G., CRAIG, M. K., CUNNINGHAM, G. E., and CONWAY, H. L., An International Mars Exploration Program, Acta Astronautica, Vol. 22, Nos. 1-12, pp. 255-267, 1990.

4. MEASE, K. D., and VINH, N. X., Minimum-Fuel Aeroassisted Coplanar Orbit Transfer Using Lift Modulation, Journal of Guidance, Control, and Dynamics, Vol. 8, No. 1, pp. 134-141, 1985.

5. MIELE, A., and VENKATARAMAN, P., Optimal Trajectories for Aeroassisted Orbital Transfer, Acta Astronautica, Vol. 11, Nos. 7-8, pp. 423-433, 1984.

6. MIELE, A., and BASAPUR, V. K., Approximate Solutions to Minimax Optimal Control Problems for Aeroassisted Orbital Transfer, Acta Astronautica, Vol. 12, No. 10, pp. 809-818, 1985.

7. MIELE, A., BASAPUR, V. K., and MEASE, K. D., Nearly-Grazing Optimal Trajectories for Aeroassisted Orbital Transfer, Journal of the Astronautical Sciences, Vol. 34, No. 1, pp. 3-18, 1986.

8. MIELE, A., BASAPUR, V. K., and LEE, W. Y., Optimal Trajectories for Aeroassisted, Coplanar Orbital Transfer, Journal of Optimization Theory and Applications, Vol. 52, No. 1, pp. $1-24,1987$. 
9. VINH, N. X., JOHANNESEN, J. R., MEASE, K. D., and HANSON, J. M., Explicit Guidance of Drag-Modulated Aeroassisted Transfer between Elliptical Orbits, Journal of Guidance, Control, and Dynamics, Vol. 9, No. 2, pp. 274-280, 1986.

10. LEE, B. S., and GRANTHAM, W. J., Aeroassisted Orbital Maneuvering Using Lyapunov Optimal Feedback Control, Journal of Guidance, Control, and Dynamics, Vol. 12, No. 2, pp. 237$242,1989$.

11. ANONYMOUS, N. N., Aeroassisted Flight Experiment: Preliminary Design Document, NASA Marshall Space Flight Center, 1986.

12. GAMBle, J. D., CERIMElE, C. J., MOORE, T. E., and HIGGINS, J., Atmospheric Guidance Concepts for an Aeroassist Flight Experiment, Journal of the Astronautical Sciences, Vol. 36, Nos. 1-2, pp. 45-71, 1988 .

13. MIELE, A., WANG, T., and LEE, W. Y., Optimization and Guidance of Trajectories for Coplanar, Aeroassisted Orbital Transfer, Rice University, Aero-Astronautics Report No. 241, 1989.

14. MIELE, A., and WANG, T., Gamma Guidance of Trajectories for Coplanar, Aeroassisted Orbital Transfer, Rice University, Aero-Astronautics Report No. 246, 1990.

15. NOAA, NASA, and USAF, US Standard Atmosphere, 1976, US Government Printing office, Washington, DC, 1976.

16. MIELE, A., WANG, T., and BASAPUR, V. K., Primal and Dual Formulations of Sequential Gradient-Restoration Algorithms for Trajectory Optimization Problems, Acta Astronautica, Vol. 13, No. 8, pp. 491-505, 1986. 
17. MIELE, A., and WANG, T., Primal-Dual Properties of Sequential Gradient-Restoration Algorithms for Optimal Control Problems, Part 1, Basic Problem, Integral Methods in Science and Engineering, Edited by F. R. Payne et al, Hemisphere Publishing Corporation, Washington, DC, pp. 577-607, 1986.

18. MIELE, A., and WANG, T., Primal-Dual Properties of Sequential Gradient-Restoration Algorithms for Optimal Control Problems, Part 2, General Problem, Journal of Mathematical Analysis and Applications, Vol. 119, Nos. 1-2, pp. 21-54, 1986. 


\section{List of Tables}

Table lA. Comparison of characteristic velocities ( $\mathrm{km} / \mathrm{sec})$, Spacecraft Sl.

Table 1B. Comparison of characteristic velocities ( $\mathrm{km} / \mathrm{sec})$, Spacecraft 52 .

Table 2A. Optimal trajectory results, spacecraft Sl. Table 2B. Optimal trajectory results, spacecraft $\mathbf{s}$. 
Table 1A. Comparison of characteristic velocities $(\mathrm{km} / \mathrm{sec})$, spacecraft Sl.

\begin{tabular}{|c|c|c|c|c|}
\hline Trajectory & $\begin{array}{l}\text { Characteristic } \\
\text { velocity }\end{array}$ & $C_{L L}=-0.90$ & $C_{L L}=-0.70$ & $C_{L I}=-0.50$ \\
\hline Grazing & $\Delta \tilde{\mathrm{V}}_{00}$ & 1.4857 & 1.4857 & 1.4857 \\
\hline Optimal & $\Delta \tilde{\mathrm{V}}_{00}$ & 1.4899 & 1.4901 & 1.4903 \\
\hline Hohmann & $\Delta \tilde{V}_{00}$ & 1.4638 & 1.4638 & 1.4638 \\
\hline Grazing & $\Delta \tilde{V}_{11}$ & 0.0615 & 0.0615 & 0.0615 \\
\hline Optimal & $\Delta \tilde{v}_{11}$ & 0.0673 & 0.0675 & 0.0678 \\
\hline Hohmann & $\Delta \tilde{V}_{11}$ & 2.4173 & 2.4173 & 2.4173 \\
\hline Grazing & $\Delta \tilde{V}$ & 1.5472 & 1.5472 & 1.5472 \\
\hline Optimal & $\Delta \tilde{V}$ & 1.5572 & 1.5576 & 1.5581 \\
\hline Hohmann & $\Delta \tilde{V}$ & 3.8810 & 3.8810 & 3.8810 \\
\hline
\end{tabular}


Table 1B. Comparison of characteristic velocities $(\mathrm{km} / \mathrm{sec})$, spacecraft $\mathrm{s} 2$.

Trajectory Characteristic $\quad C_{L L}=-0.47 \quad C_{L L}=-0.38 \quad C_{L L}=-0.27$
velocity

\begin{tabular}{lcccc}
\hline Grazing & $\Delta \tilde{\mathrm{V}}_{00}$ & 1.4857 & 1.4857 & 1.4857 \\
Optimal & $\Delta \tilde{\mathrm{V}}_{00}$ & 1.4896 & 1.4897 & 1.4899 \\
Hohmann & $\Delta \tilde{\mathrm{V}}_{00}$ & 1.4638 & 1.4638 & 1.4638 \\
\hline Grazing & $\Delta \tilde{\mathrm{V}}_{11}$ & 0.0615 & 0.0615 & 0.0615 \\
Optimal & $\Delta \tilde{\mathrm{V}}_{11}$ & 0.0684 & 0.0702 & 0.0733 \\
Hohmann & $\Delta \tilde{\mathrm{V}}_{11}$ & 2.4173 & 2.4173 & 2.4173 \\
\hline Grazing & $\Delta \tilde{\mathrm{V}}$ & 1.5472 & 1.5472 & 1.5472 \\
Optimal & $\Delta \tilde{\mathrm{V}}$ & 1.5580 & 1.5599 & 1.5632 \\
Hohmann & $\Delta \tilde{\mathrm{V}}$ & 3.8810 & 3.8810 & 3.8810 \\
\hline
\end{tabular}


Table 2A. Optimal trajectory results, spacecraft Sl.

\begin{tabular}{|c|c|c|c|c|}
\hline Quantity & $C_{L I}=-0.90$ & $C_{L I}=-0.70$ & $C_{L L}=-0.50$ & Units \\
\hline $\mathrm{h}_{0}$ & 120.00 & 120.00 & 120.00 & $\mathrm{~km}$ \\
\hline$v_{0}$ & 9.8371 & 9.8371 & 9.8372 & $\mathrm{~km} / \mathrm{sec}$ \\
\hline$\tilde{\mathrm{v}}_{0}$ & 10.3097 & 10.3096 & 10.3096 & $\mathrm{~km} / \mathrm{sec}$ \\
\hline$\gamma_{0}$ & -4.297 & -4.388 & -4.508 & deg \\
\hline$\tilde{\gamma}_{0}$ & -4.100 & -4.187 & -4.301 & deg \\
\hline $\mathrm{h}_{1}$ & 120.00 & 120.00 & 120.00 & $\mathrm{~km}$ \\
\hline $\mathrm{V}_{1}$ & 7.4146 & 7.4145 & 7.4141 & $\mathrm{~km} / \mathrm{sec}$ \\
\hline$\tilde{\mathrm{v}}_{1}$ & 7.8884 & 7.8883 & 7.8880 & $\mathrm{~km} / \mathrm{sec}$ \\
\hline$\gamma_{1}$ & 0.592 & 0.601 & 0.616 & deg \\
\hline$\tilde{\gamma}_{1}$ & 0.557 & 0.565 & .0 .579 & deg \\
\hline $\min (h)$ & 75.35 & 73.46 & 70.96 & $\mathrm{~km}$ \\
\hline $\max (D P)$ & 1675 & 2245 & 3264 & $\mathrm{~N} / \mathrm{m}^{2}$ \\
\hline $\max (H R)$ & 177.1 & 206.3 & 251.2 & $w / \mathrm{cm}^{2}$ \\
\hline$\tau$ & 1872.2 & 2172.1 & 2547.6 & sec \\
\hline$\Delta \tilde{\mathrm{V}}_{00}$ & 1.4899 & 1.4901 & 1.4903 & $\mathrm{~km} / \mathrm{sec}$ \\
\hline$\Delta \tilde{\mathrm{V}}_{11}$ & 0.0673 & 0.0675 & 0.0678 & $\mathrm{~km} / \mathrm{sec}$ \\
\hline$\Delta \tilde{V}$ & 1.5572 & 1.5576 & 1.5581 & $\mathrm{~km} / \mathrm{sec}$ \\
\hline
\end{tabular}


Table 2B. Optimal trajectory results, spacecraft $\mathbf{S 2}$.

\begin{tabular}{|c|c|c|c|c|}
\hline Quantity & $C_{L L}=-0.47$ & $C_{L L}=-0.38$ & $C_{L L}=-0.27$ & Units \\
\hline$h_{0}$ & 120.00 & 120.00 & 120.00 & $\mathrm{~km}$ \\
\hline$v_{0}$ & 9.8371 & 9.8371 & 9.8371 & $\mathrm{~km} / \mathrm{sec}$ \\
\hline$\tilde{\mathrm{v}}_{0}$ & 10.3097 & 10.3097 & 10.3097 & $\mathrm{~km} / \mathrm{sec}$ \\
\hline$\gamma_{0}$ & -4.167 & -4.215 & -4.293 & deg \\
\hline$\tilde{\gamma}_{0}$ & -3.976 & -4.022 & -4.096 & deg \\
\hline $\mathrm{h}_{1}$ & 120.00 & 120.00 & 120.00 & $\mathrm{~km}$ \\
\hline $\mathrm{v}_{1}$ & 7.4136 & 7.4119 & 7.4089 & $\mathrm{~km} / \mathrm{sec}$ \\
\hline$\tilde{\mathrm{v}}_{1}$ & 7.8874 & 7.8857 & 7.8827 & $\mathrm{~km} / \mathrm{sec}$ \\
\hline$\gamma_{1}$ & 0.644 & 0.721 & 0.841 & deg \\
\hline$\tilde{Y}_{1}$ & 0.605 & 0.678 & 0.790 & deg \\
\hline $\min (h)$ & 78.52 & 78.09 & 77.25 & $\mathrm{~km}$ \\
\hline $\max (D P)$ & 986 & 1034 & 1153 & $\mathrm{~N} / \mathrm{m}^{2}$ \\
\hline $\max (H R)$ & 131.6 & 132.7 & 138.2 & $\mathrm{w} / \mathrm{cm}^{2}$ \\
\hline$\tau$ & 1069.1 & 856.4 & 691.2 & sec \\
\hline$\Delta \tilde{\mathrm{V}}_{00}$ & 1.4896 & 1.4897 & 1.4899 & $\mathrm{~km} / \mathrm{sec}$ \\
\hline$\Delta \tilde{\mathrm{V}}_{11}$ & 0.0684 & 0.0702 & 0.0733 & $\mathrm{~km} / \mathrm{sec}$ \\
\hline$\Delta \tilde{\mathrm{V}}$ & 1.5580 & 1.5599 & 1.5632 & $\mathrm{~km} / \mathrm{sec}$ \\
\hline
\end{tabular}




\section{List of Figures}

Fig. 1. Coplanar, aeroassisted orbital transfer.

Fig. 2A. Drag polar, Spacecraft Sl.

Fig. 2B. Drag polar, Spacecraft S2.

Fig. 3A. Optimal trajectories, altitude vs time, spacecraft sl.

Fig. 3B. Optimal trajectories, velocity vs time, Spacecraft SI.

Fig. 3C. Optimal trajectories, path inclination vs time, Spacecraft Sl.

Fig. 3D. Optimal trajectories, lift coefficient vs time, Spacecraft SI.

Fig. 3E. Optimal trajectories, density vs time, spacecraft $\mathrm{Sl}$.

Fig. 4A. Optimal trajectories, altitude vs time, spacecraft $\mathrm{S} 2$.

Fig. 4B. Optimal trajectories, velocity vs time, Spacecraft $\mathrm{s} 2$.

Fig. 4C. Optimal trajectories, path inclination vs time, spacecraft $\mathrm{s} 2$.

Fig. 4D. Optimal trajectories, lift coefficient vs time, spacecraft S2.

Fig. 4E. Optimal trajectories, density vs time, spacecraft S2. 


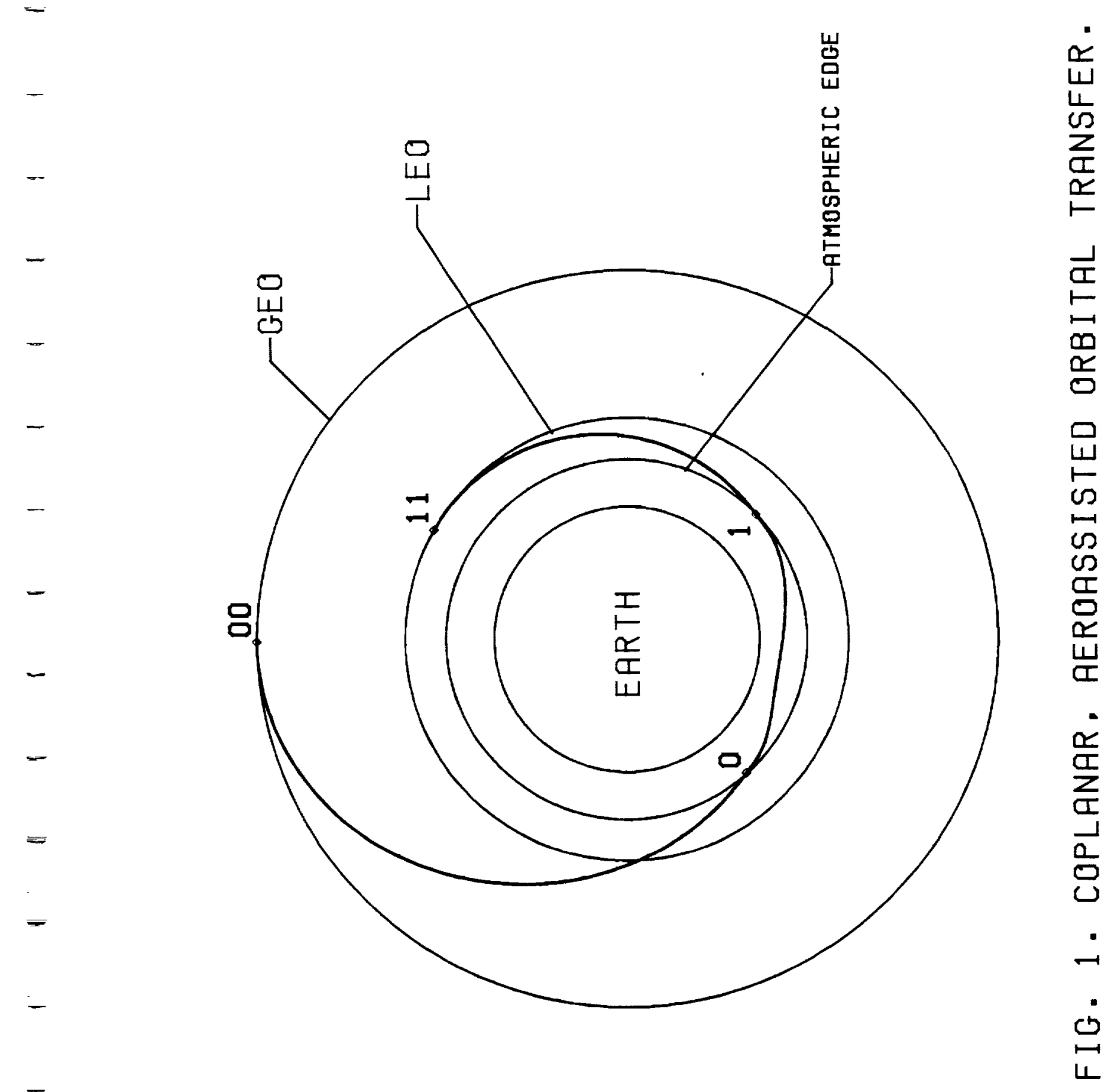



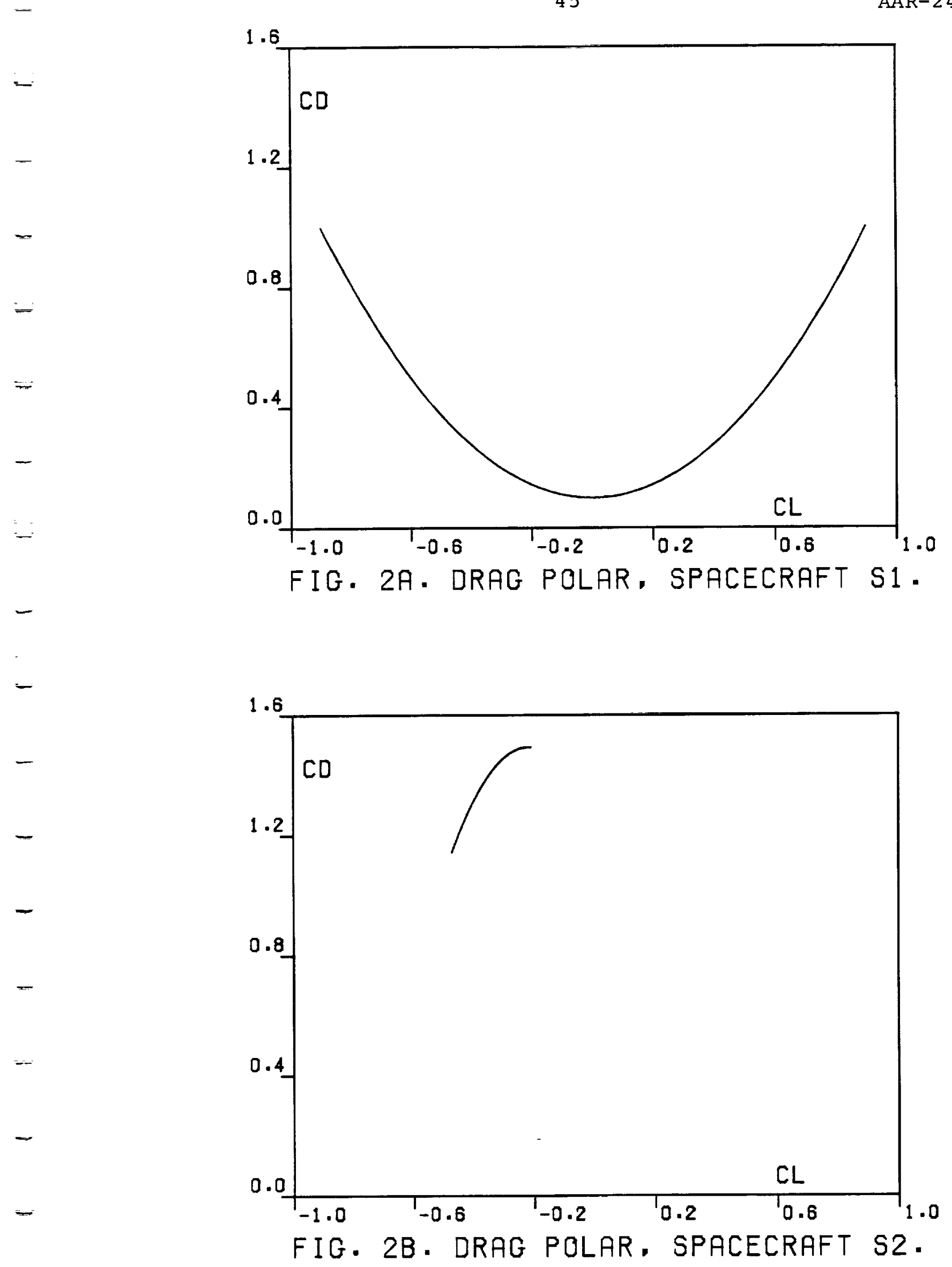

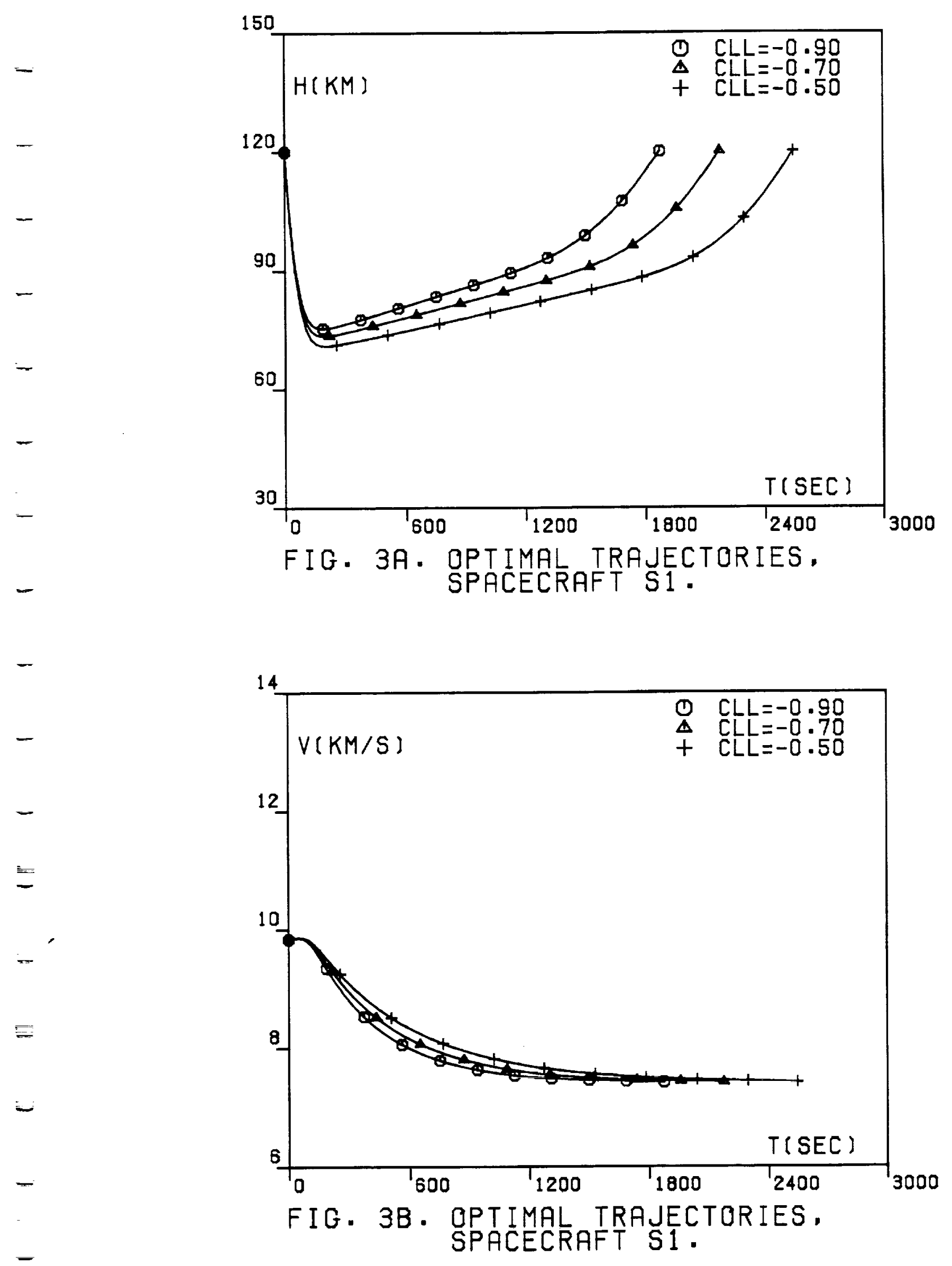


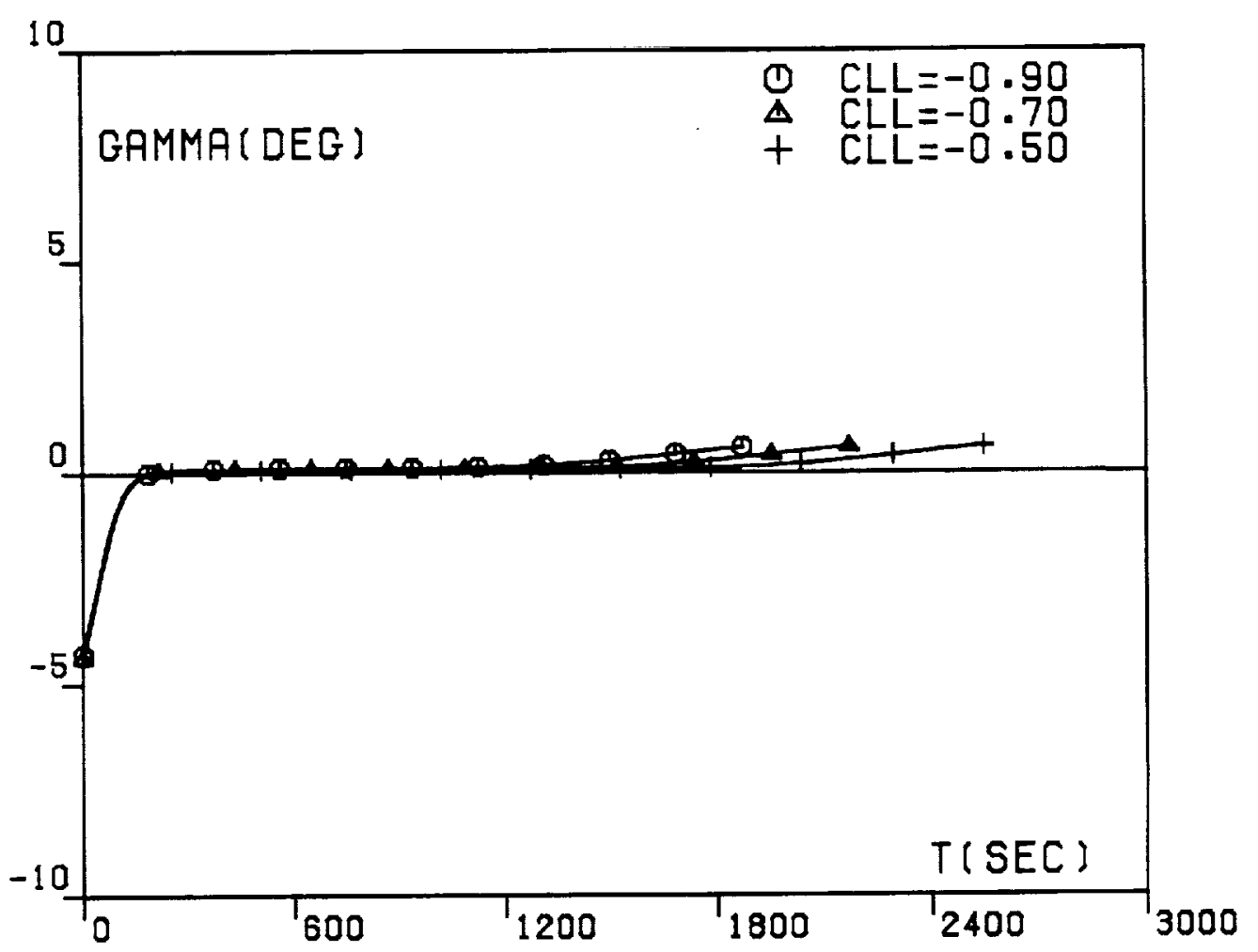

FIG. 3C . GPTIMAL TRAJECTORIES,

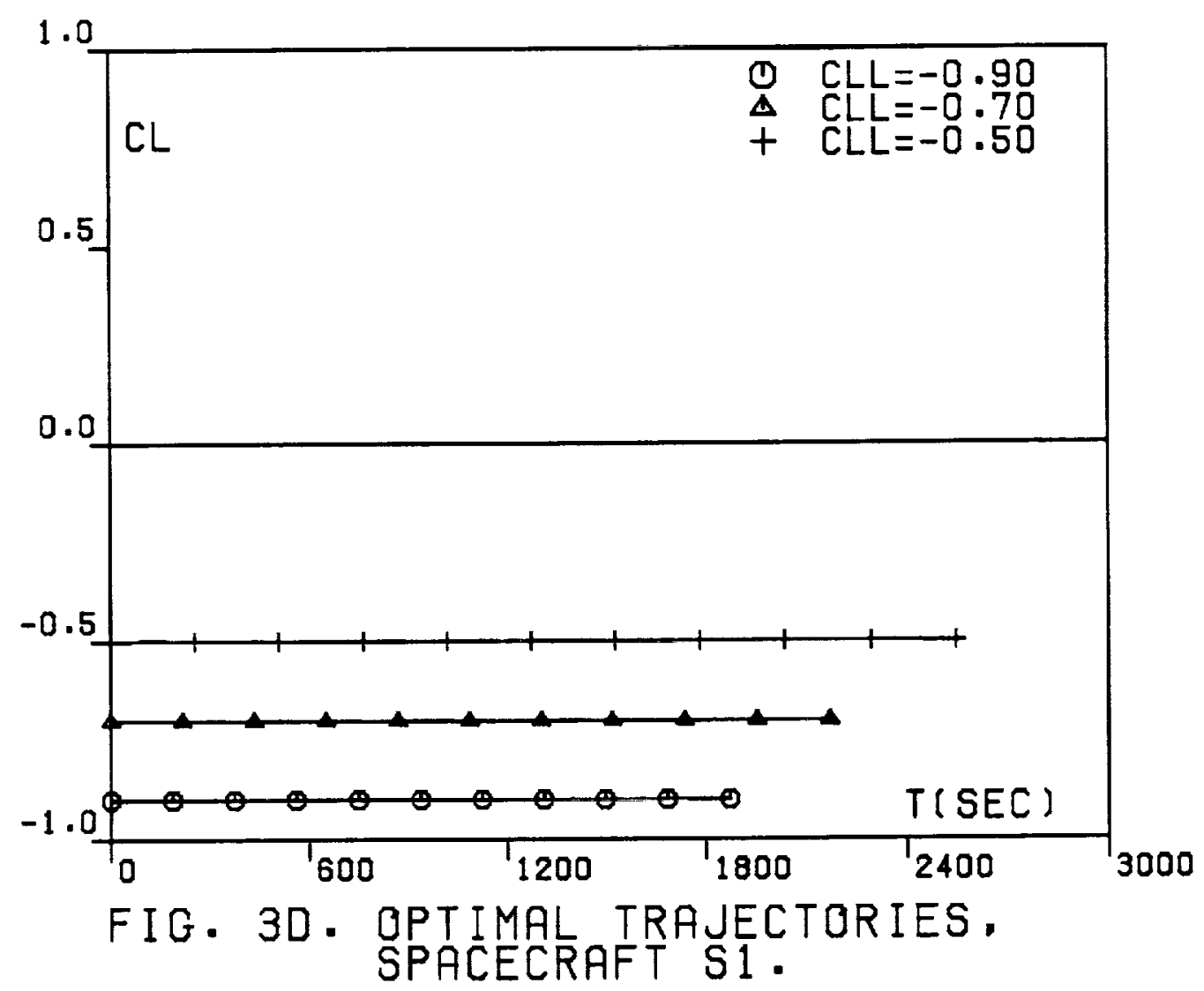




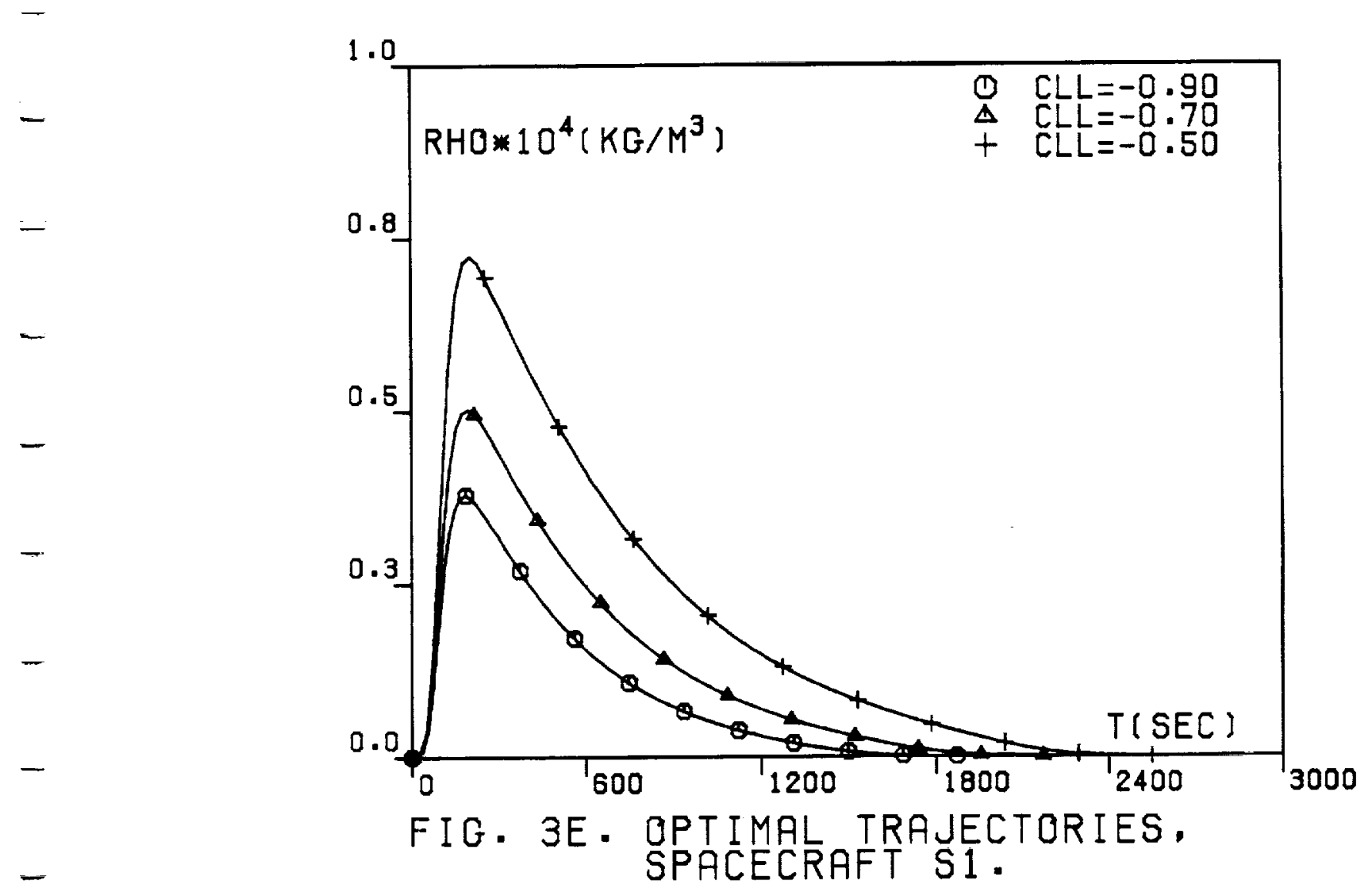

$-$

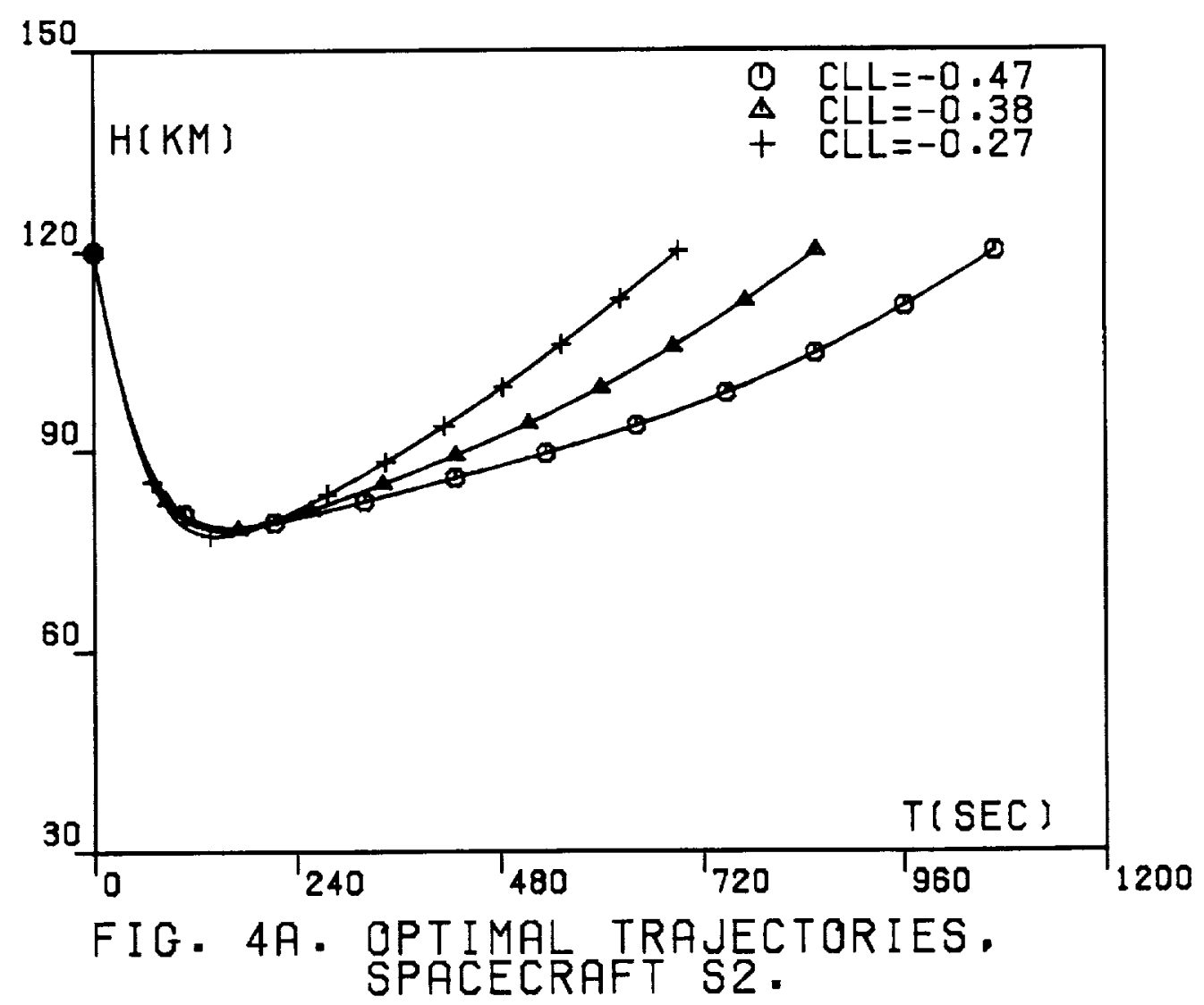



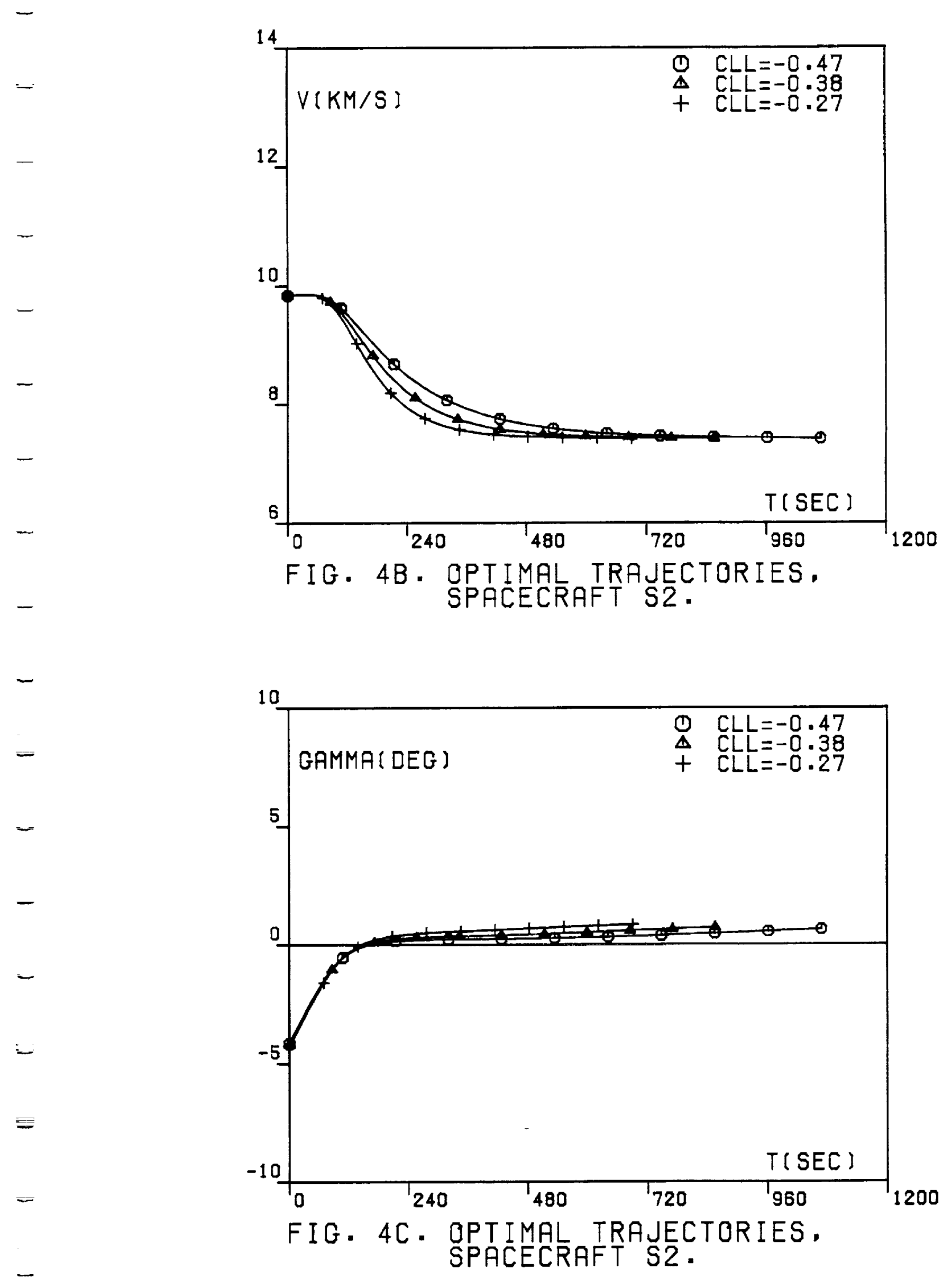


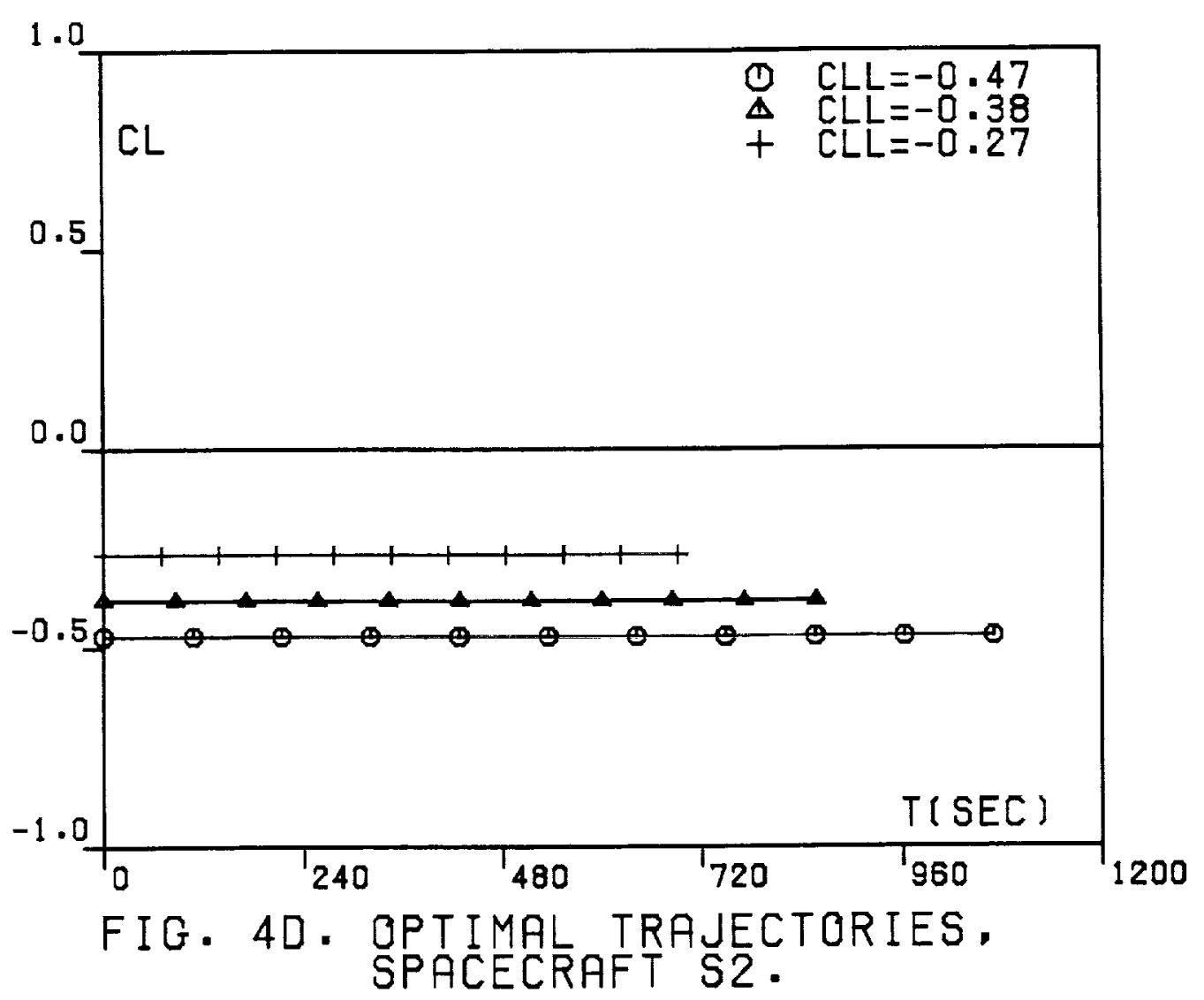

-

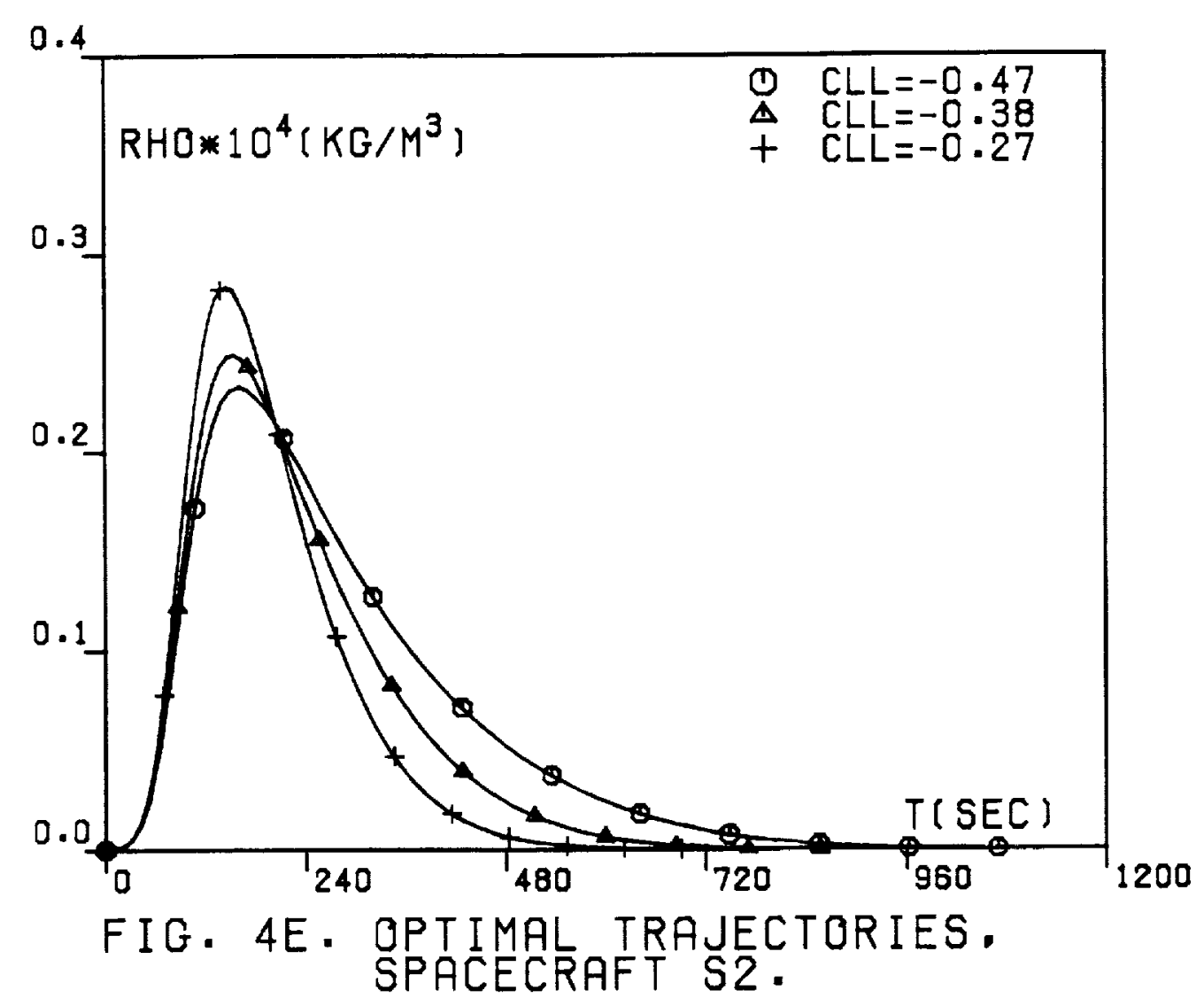

